INTERNATIONAL MONETARY FUND

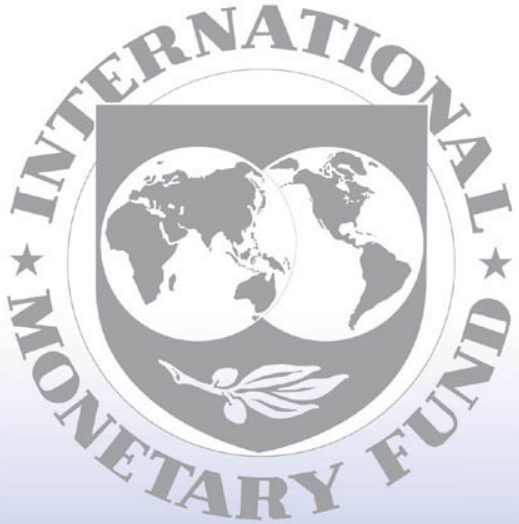

Staff

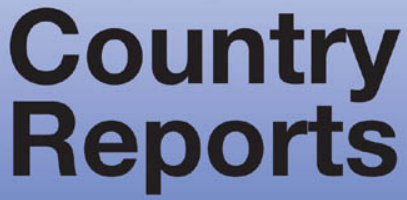




\section{Federated States of Micronesia: Statistical Appendix}

This Statistical Appendix for the Federated States of Micronesia was prepared by a staff team of the International Monetary Fund as background documentation for the periodic consultation with the member country. It is based on the information available at the time it was completed on February 10, 2009. The views expressed in this document are those of the staff team and do not necessarily reflect the views of the government of the Federated States of Micronesia or the Executive Board of the IMF.

The policy of publication of staff reports and other documents by the IMF allows for the deletion of market-sensitive information.

Copies of this report are available to the public from

International Monetary Fund • Publication Services

700 19th Street, N.W. • Washington, D.C. 20431

Telephone: (202) 623-7430 • Telefax: (202) 623-7201

E-mail: publications@imf.org • Internet: http://www.imf.org

\section{International Monetary Fund Washington, D.C.}


This page intentionally left blank 


\section{INTERNATIONAL MONETARY FUND \\ FEDERATED STATES OF MICRONESIA}

\section{Statistical Appendix}

Prepared by Martin Sommer and Ioana Hussiada (both APD)

Approved by the Asia and Pacific Department

February 10, 2009

Contents

Page

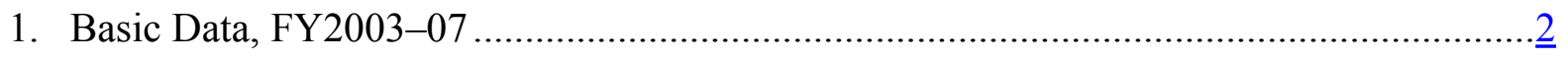

2. Gross Domestic Product, FY2003-07 ......................................................................... $\underline{3}$

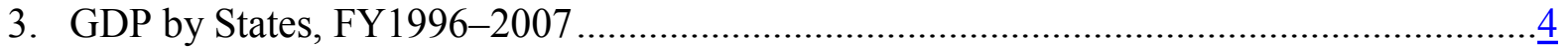

4. Public and Private Sector Employment and Wages, FY2001-07.................................. $\underline{5}$

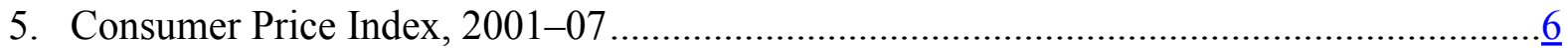

6. Pohnpei Consumer Price Index, 2001-07 .............................................................

7. Chuuk Consumer Price Index, 2001-07 ......................................................................... $\underline{8}$

8. Kosrae Consumer Price Index, 2001-07 ....................................................................

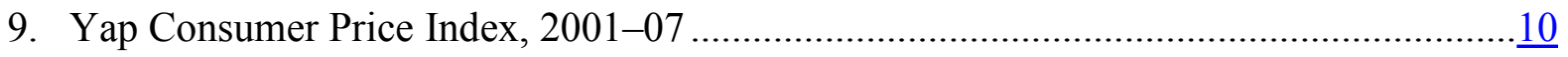

10. Consolidated General Government Finances, FY2002-07 ........................................11

11. National Government Finances, FY2002-07 .............................................................

12. Pohnpei Government Finances, FY02-07 ..............................................................13

13. Kosrae Government Finances, FY2002-07 ............................................................. 14

14. Yap Government Finances, FY2002-07..................................................................... 15

15. Chuuk Government Finances, FY2002-07..........................................................16

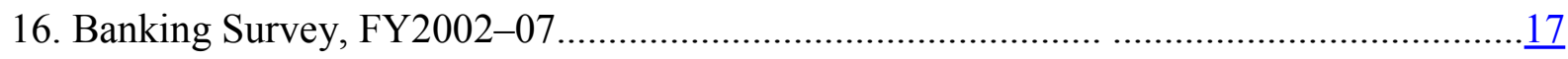

17. Interest Rates of Deposit Money Banks, 2001-08..................................................... 18

18. Balance of Payments, FY2002-06..................................................................... 19

19. External Debt and Debt Service Obligations, FY2001-07 ..........................................20 
Table 1. Federated States of Micronesia: Basic Data, FY2003-07 1/

$\begin{array}{ll}\text { Nominal GDP (FY2007): } & \text { US } \$ 238 \text { million } \\ \text { Population (FY2007): } & 108,031 \\ \text { GDP per capita (FY2007): } & \text { US } \$ 2,204 \\ \text { IMF Quota: } & \text { SDR5.1 million }\end{array}$

\begin{tabular}{|c|c|c|c|c|c|}
\hline & FY2003 & FY2004 & FY2005 & FY2006 & $\begin{array}{c}\text { FY2007 } \\
\text { Est. }\end{array}$ \\
\hline \multicolumn{6}{|c|}{ Real sector (average annual percent change unless otherwise noted) } \\
\hline Real GDP & 2.9 & -3.3 & -0.5 & -1.6 & -3.6 \\
\hline Consumer prices & -0.2 & 1.8 & 4.1 & 4.4 & 3.6 \\
\hline Employment & -1.9 & -0.9 & -1.1 & 1.5 & -0.7 \\
\hline Public (incl. public enterprises) & -2.7 & -2.8 & 0.0 & 2.9 & -5.9 \\
\hline Private & -0.9 & 1.5 & -2.1 & 0.1 & 5.5 \\
\hline Nominal wages & 2.1 & -0.7 & 1.6 & 1.5 & -1.1 \\
\hline Public-private wage ratio & 2.1 & 2.1 & 2.2 & 2.1 & 2.2 \\
\hline \multicolumn{6}{|c|}{ Consolidated government finance (in percent of GDP) } \\
\hline Revenue and grants & 74.8 & 59.6 & 58.6 & 58.6 & 61.0 \\
\hline Revenue & 21.2 & 26.7 & 22.4 & 23.0 & 22.2 \\
\hline Grants & 53.6 & 33.0 & 36.1 & 35.6 & 38.8 \\
\hline Expenditure & 72.0 & 75.6 & 63.9 & 65.0 & 64.3 \\
\hline Current & 60.7 & 56.6 & 58.9 & 62.3 & 58.7 \\
\hline Capital & 11.3 & 19.0 & 4.9 & 2.8 & 5.5 \\
\hline Overall balance & 2.8 & -16.0 & -5.3 & -6.4 & -3.3 \\
\hline Compact Trust Fund (millions of U.S. dollars) & 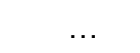 & 62.5 & 80.4 & 99.0 & 122.5 \\
\hline \multicolumn{6}{|c|}{ Commercial banks (in millions of U.S. dollars; end of period) } \\
\hline Foreign assets & 110.0 & 105.2 & 99.2 & 101.1 & 106.2 \\
\hline Loans & 23.7 & 21.3 & 25.7 & 30.0 & 35.3 \\
\hline Total deposits & 119.8 & 115.4 & 111.4 & 113.7 & 119.5 \\
\hline \multicolumn{6}{|l|}{ Interest rates (in percent, average for $\mathrm{FY}$ ) } \\
\hline Consumer loans & 15.1 & 15.1 & 16.4 & 15.8 & 14.3 \\
\hline Commercial loans & 6.9 & 6.9 & 7.8 & 8.6 & 9.0 \\
\hline \multicolumn{6}{|l|}{ Balance of payments (in millions of U.S. dollars) } \\
\hline Trade balance & -96.2 & -120.3 & -122.2 & -128.9 & -127.5 \\
\hline Net services and income & -27.1 & -23.5 & -22.1 & -22.1 & -19.5 \\
\hline Private and official transfers & 123.0 & 99.1 & 115.2 & 112.7 & 116.6 \\
\hline Current account including official transfers & -0.4 & -44.7 & -29.1 & -38.4 & -30.3 \\
\hline (in percent of GDP) & -0.2 & -20.0 & -12.5 & -16.1 & -12.7 \\
\hline Current account excluding official transfers & -121.0 & -141.3 & -140.9 & -145.7 & -141.1 \\
\hline (in percent of GDP) & -53.1 & -63.1 & -60.7 & -61.1 & -59.2 \\
\hline $\begin{array}{l}\text { Overall balance } \\
\text { (in percent of GDP) }\end{array}$ & $\begin{array}{r}16.6 \\
7.3\end{array}$ & $\begin{array}{l}-58.6 \\
-26.2\end{array}$ & $\begin{array}{l}-8.9 \\
-3.8\end{array}$ & $\begin{array}{r}-19.5 \\
-8.2\end{array}$ & $\begin{array}{l}-7.1 \\
-3.0\end{array}$ \\
\hline Gross reserves (in months of imports) & 6.3 & 3.5 & 3.1 & 2.8 & 2.9 \\
\hline \multicolumn{6}{|c|}{ External debt (in millions of U.S. dollars; end of period) $2 /$} \\
\hline Stock & 62.7 & 62.9 & 64.0 & 65.8 & 67.2 \\
\hline (in percent of GDP) & 27.5 & 28.1 & 27.6 & 27.6 & 28.2 \\
\hline Debt service & 2.5 & 2.6 & 2.5 & 3.4 & 2.8 \\
\hline (in percent of exports of goods and services) & 5.7 & 7.4 & 6.7 & 9.1 & 6.4 \\
\hline \multicolumn{2}{|l|}{ Exchange rate regime } & \multicolumn{4}{|c|}{ U.S. dollar is the official currency } \\
\hline Real effective exchange rate $3 /$ & 98.2 & 95.4 & 95.6 & 98.3 & 99.6 \\
\hline
\end{tabular}

Sources: Data provided by the FSM authorities and Fund staff estimates.

$1 /$ Fiscal year ending September 30.

2/ Government and public enterprise debt only.

$3 /$ Year $2000=100$. 
Table 2. Federated States of Micronesia: Gross Domestic Product, FY2003-07 1/

\begin{tabular}{|c|c|c|c|c|c|}
\hline & FY2003 & FY2004 & FY2005 & FY2006 & $\begin{array}{r}\text { FY2007 } \\
\text { Est. }\end{array}$ \\
\hline & \multicolumn{5}{|c|}{ (In millions of U.S. dollars) } \\
\hline Productive enterprises & 82.0 & 80.4 & 80.7 & 80.9 & 83.3 \\
\hline Private sector & 64.0 & 63.4 & 64.2 & 65.0 & 67.6 \\
\hline Public enterprises & 18.0 & 17.0 & 16.5 & 16.0 & 15.7 \\
\hline Financial enterprises & 4.3 & 4.8 & 5.5 & 6.4 & 8.5 \\
\hline Government & 69.8 & 68.5 & 70.5 & 72.8 & 68.7 \\
\hline Non-profit organizations & 2.5 & 2.7 & 2.8 & 2.8 & 2.9 \\
\hline Households & 54.9 & 54.2 & 57.5 & 60.7 & 63.2 \\
\hline Subsistence & 33.3 & 32.4 & 34.6 & 36.9 & 38.4 \\
\hline Home ownership & 21.5 & 21.9 & 22.9 & 23.9 & 24.7 \\
\hline Indirect taxes & 19.1 & 18.5 & 21.1 & 21.7 & 21.0 \\
\hline less bank service charge & -4.8 & -4.9 & -6.1 & -7.2 & -9.6 \\
\hline \multirow[t]{2}{*}{ Nominal GDP } & 227.7 & 224.1 & 232.1 & 238.3 & 238.1 \\
\hline & \multicolumn{5}{|c|}{ (In percent of GDP) } \\
\hline Productive enterprises & 36.0 & 35.9 & 34.8 & 34.0 & 35.0 \\
\hline Financial enterprises & 1.9 & 2.1 & 2.4 & 2.7 & 3.6 \\
\hline Government & 30.6 & 30.5 & 30.4 & 30.6 & 28.8 \\
\hline Nonprofit organizations & 1.1 & 1.2 & 1.2 & 1.2 & 1.2 \\
\hline Households & 24.1 & 24.2 & 24.8 & 25.5 & 26.5 \\
\hline \multicolumn{6}{|l|}{ Memorandum items: } \\
\hline Nominal GDP per capita (in U.S. dollars) & 2,115 & 2,079 & 2,151 & 2,207 & 2,204 \\
\hline Real GDP growth rate (in percent) & 2.9 & -3.3 & -0.5 & -1.6 & -3.6 \\
\hline Real GDP (in FY98 millions U.S. dollars) & 217.0 & 209.8 & 208.7 & 205.3 & 197.9 \\
\hline
\end{tabular}

Sources: Data provided by the FSM authorities; and Fund staff estimates.

1/ Fiscal year ending September 30. 
Table 3. Federated States of Micronesia: GDP by States, FY1996-2007 1/

(In millions of U.S. dollars)

\begin{tabular}{|c|c|c|c|c|c|c|c|c|c|c|c|c|}
\hline & FY1996 & FY1997 & FY1998 & FY1999 & FY2000 & FY2001 & FY2002 & FY2003 & FY2004 & FY2005 & $\begin{array}{r}\text { FY2006 } \\
\text { Est. } \\
\end{array}$ & $\begin{array}{r}\text { FY2007 } \\
\text { Est. }\end{array}$ \\
\hline \multicolumn{13}{|l|}{ Pohnpei 2/ } \\
\hline Nominal GDP & 95.7 & 87.7 & 92.1 & 91.5 & 95.2 & 95.8 & 100.0 & 100.9 & 102.4 & 105.8 & 109.8 & 113.0 \\
\hline Real GDP 3/ & 99.9 & 89.1 & 92.1 & 89.8 & 90.0 & 89.4 & 94.3 & 95.4 & 95.9 & 94.6 & 95.2 & 95.1 \\
\hline Percent change & -2.2 & -10.8 & 3.3 & -2.5 & 0.3 & -0.7 & 5.4 & 1.2 & 0.5 & -1.4 & 0.7 & -0.1 \\
\hline \multicolumn{13}{|l|}{ Chuuk } \\
\hline Nominal GDP & 59.9 & 52.4 & 57.3 & 58.2 & 66.7 & 66.6 & 66.2 & 68.2 & 66.5 & 67.0 & 69.4 & 65.9 \\
\hline Real GDP 3/ & 62.5 & 53.3 & 57.3 & 57.1 & 64.5 & 63.8 & 62.8 & 64.6 & 60.8 & 59.4 & 58.4 & 53.3 \\
\hline Percent change & -9.4 & -14.8 & 7.7 & -0.4 & 12.9 & -1.1 & -1.6 & 2.9 & -5.8 & -2.3 & -1.7 & -8.8 \\
\hline \multicolumn{13}{|l|}{ Kosrae } \\
\hline Nominal GDP & 17.9 & 16.5 & 15.5 & 17.0 & 17.4 & 18.6 & 18.9 & 18.1 & 17.7 & 18.1 & 18.2 & 18.6 \\
\hline Real GDP 3/ & 18.7 & 16.8 & 15.5 & 16.7 & 16.8 & 18.0 & 18.8 & 18.4 & 17.5 & 16.5 & 15.9 & 16.0 \\
\hline Percent change & 2.3 & -10.4 & -7.3 & 7.6 & 0.7 & 6.7 & 4.9 & -2.4 & -4.6 & -6.0 & -3.3 & 0.3 \\
\hline \multicolumn{13}{|l|}{ Yap } \\
\hline Nominal GDP & 33.4 & 33.5 & 38.7 & 36.5 & 37.9 & 39.2 & 36.8 & 40.5 & 37.5 & 41.2 & 40.9 & 40.6 \\
\hline Real GDP 3/ & 34.9 & 34.1 & 38.7 & 35.8 & 37.0 & 37.3 & 35.0 & 38.8 & 36.3 & 38.6 & 36.5 & 34.7 \\
\hline Percent change & 4.2 & -2.3 & 13.8 & -7.6 & 3.2 & 0.9 & -6.1 & 10.9 & -6.4 & 6.3 & -5.4 & -5.0 \\
\hline \multicolumn{13}{|l|}{ Micronesia total } \\
\hline Nominal GDP & 207.0 & 190.1 & 203.7 & 203.2 & 217.1 & 220.2 & 221.9 & 227.7 & 224.1 & 232.1 & 238.3 & 238.1 \\
\hline Real GDP 3/ & 216.0 & 193.2 & 203.7 & 199.4 & 208.8 & 209.0 & 210.9 & 217.0 & 209.8 & 208.7 & 205.3 & 197.9 \\
\hline Percent change & -3.1 & -10.6 & 5.4 & -2.1 & 4.7 & 0.1 & 0.9 & 2.9 & -3.3 & -0.5 & -1.6 & -3.6 \\
\hline
\end{tabular}

Sources: Data provided by the FSM authorities; and Fund staff estimates.

1/ Fiscal year ending September 30. Real GDP estimates are at FY1998 prices.

2/ Includes national government.

3/ U.S. CPI is used through FY1999 and FSM CPI for FY2000 and onwards. 
Table 4. Federated States of Micronesia: Public and Private Sector Employment and Wages, FY2001-07 1/

\begin{tabular}{|c|c|c|c|c|c|c|c|}
\hline & FY2001 & FY2002 & FY2003 & FY2004 & FY2005 & FY2006 & FY2007 \\
\hline & \multicolumn{7}{|c|}{ (In persons) } \\
\hline Total employment & 16,675 & 16,874 & 16,555 & 16,406 & 16,220 & 16,470 & 16,360 \\
\hline Total public sector & 7,374 & 7,751 & 7,418 & 7,082 & 7,146 & 7,795 & 7,237 \\
\hline National and state government & 5,757 & 5,912 & 5,668 & 5,614 & 5,889 & 6,524 & 6,015 \\
\hline National government & 839 & 826 & 854 & 851 & 655 & 665 & 663 \\
\hline Pohnpei & 1,698 & 1,748 & 1,721 & 1,712 & 1,750 & 1,876 & 1,910 \\
\hline Chuuk & 2,603 & 2,669 & 2,421 & 2,380 & 2,364 & 2,830 & 2,355 \\
\hline Yap & 781 & 800 & 819 & 814 & 1,049 & 1,085 & 1,039 \\
\hline Kosrae & 675 & 695 & 706 & 709 & 727 & 733 & 711 \\
\hline Municipalities & 706 & 959 & 872 & 603 & 497 & 585 & 556 \\
\hline Public enterprises & 910 & 881 & 878 & 865 & 760 & 687 & 667 \\
\hline Private sector & 7,281 & 7,036 & 7,001 & 7,103 & 6,939 & 6,941 & 7,326 \\
\hline Private Nonfinancial enterprises & 6,544 & 6,275 & 6,274 & 6,384 & 6,240 & 6,238 & 6,602 \\
\hline \multicolumn{8}{|l|}{ Of which: } \\
\hline Wholesale and retail trade & 2,721 & 2,798 & 2,927 & 3,085 & 3,238 & 3,338 & 3,451 \\
\hline Transport & 1,007 & 964 & 1,056 & 1,026 & 1,055 & 1,100 & 1,144 \\
\hline Education & 925 & 1,070 & 1,118 & 1,180 & 1,115 & 872 & 928 \\
\hline Construction & 1,115 & 895 & 808 & 838 & 856 & 814 & 813 \\
\hline Hotels and restaurants & 890 & 796 & 799 & 838 & 849 & 821 & 865 \\
\hline Manufacturing & 822 & 796 & 682 & 591 & 186 & 92 & 110 \\
\hline Financial institutions & 207 & 201 & 175 & 177 & 188 & 195 & 198 \\
\hline \multirow{2}{*}{ Nonprofit organizations } & 530 & 560 & 552 & 542 & 511 & 508 & 526 \\
\hline & \multicolumn{7}{|c|}{ (In U.S. dollars) } \\
\hline \multicolumn{8}{|l|}{ Average public sector wage } \\
\hline National and state government & 9,873 & 10,292 & 10,330 & 10,193 & 11,103 & 11,081 & 11,183 \\
\hline Municipalities & 3,572 & 3,289 & 3,386 & 3,236 & 4,813 & 4,985 & 3,500 \\
\hline Public enterprises & 10,502 & 10,435 & 10,583 & 10,823 & 11,336 & 12,276 & 12,608 \\
\hline Average private sector wage & 3,954 & 4,021 & 4,118 & 4,057 & 4,062 & 4,123 & 4,074 \\
\hline Financial enterprises & 15,549 & 15,376 & 16,129 & 13,970 & 13,539 & 14,044 & 18,351 \\
\hline Nonprofit organizations & 4,253 & 4,333 & 4,365 & 4,703 & 5,136 & 5,328 & 5,340 \\
\hline \multicolumn{8}{|l|}{ Memorandum item: } \\
\hline Total population & 107,263 & 107,472 & 107,644 & 107,785 & 107,885 & 107,965 & 108,031 \\
\hline
\end{tabular}

Sources: Data provided by the FSM authorities; and Fund staff estimates.

1/ Fiscal year ending September 30. 
Table 5. Federated States of Micronesia: Consumer Price Index, 2001-07

\begin{tabular}{|c|c|c|c|c|c|c|c|c|c|}
\hline & & Total & Food & $\begin{array}{c}\text { Tobacco, } \\
\text { Alcohol, } \\
\text { Betelnut, } \\
\text { Sakau }\end{array}$ & $\begin{array}{l}\text { Clothing, } \\
\text { Footwear }\end{array}$ & Housing & $\begin{array}{l}\text { Fuel, Light \& } \\
\text { Water }\end{array}$ & Services & Miscellaneous \\
\hline & & \multicolumn{8}{|c|}{$(2000=100)$} \\
\hline 2001 & $\begin{array}{l}\text { Qtr } 1 \\
\text { Qtr } 2 \\
\text { Qtr } 3 \\
\text { Qtr } 4\end{array}$ & $\begin{array}{l}102.8 \\
102.6 \\
102.5 \\
102.7\end{array}$ & $\begin{array}{l}100.6 \\
100.3 \\
100.4 \\
100.9\end{array}$ & $\begin{array}{l}103.4 \\
103.7 \\
105.4 \\
101.8\end{array}$ & $\begin{array}{l}96.8 \\
96.6 \\
96.6 \\
96.2\end{array}$ & $\begin{array}{c}99.6 \\
100.1 \\
98.7 \\
98.5\end{array}$ & $\begin{array}{l}112.6 \\
111.2 \\
113.2 \\
119.2\end{array}$ & $\begin{array}{l}110.5 \\
110.5 \\
111.8 \\
112.5\end{array}$ & $\begin{array}{l}99.7 \\
99.5 \\
95.9 \\
95.4\end{array}$ \\
\hline 2002 & $\begin{array}{l}\text { Qtr } 1 \\
\text { Qtr } 2 \\
\text { Qtr } 3 \\
\text { Qtr } 4\end{array}$ & $\begin{array}{l}102.8 \\
102.7 \\
102.4 \\
102.3\end{array}$ & $\begin{array}{c}101.7 \\
101.2 \\
100.7 \\
99.8\end{array}$ & $\begin{array}{c}97.2 \\
99.7 \\
99.7 \\
100.9\end{array}$ & $\begin{array}{l}97.0 \\
96.5 \\
95.7 \\
95.4\end{array}$ & $\begin{array}{l}99.8 \\
99.1 \\
98.6 \\
99.3\end{array}$ & $\begin{array}{l}123.2 \\
120.5 \\
120.5 \\
123.6\end{array}$ & $\begin{array}{l}111.8 \\
112.4 \\
112.0 \\
112.1\end{array}$ & $\begin{array}{l}95.4 \\
95.4 \\
95.3 \\
95.4\end{array}$ \\
\hline 2003 & $\begin{array}{l}\text { Qtr } 1 \\
\text { Qtr } 2 \\
\text { Qtr } 3 \\
\text { Qtr } 4\end{array}$ & $\begin{array}{l}102.0 \\
102.6 \\
102.7 \\
103.5\end{array}$ & $\begin{array}{c}99.3 \\
100.5 \\
100.4 \\
101.0\end{array}$ & $\begin{array}{l}98.9 \\
97.9 \\
98.3 \\
97.4\end{array}$ & $\begin{array}{l}95.9 \\
95.9 \\
97.0 \\
97.0\end{array}$ & $\begin{array}{l}97.1 \\
97.0 \\
96.6 \\
96.7\end{array}$ & $\begin{array}{l}125.0 \\
124.9 \\
126.7 \\
134.4\end{array}$ & $\begin{array}{l}112.5 \\
113.3 \\
113.1 \\
113.4\end{array}$ & $\begin{array}{l}95.5 \\
95.7 \\
95.8 \\
96.1\end{array}$ \\
\hline 2004 & $\begin{array}{l}\text { Qtr } 1 \\
\text { Qtr } 2 \\
\text { Qtr } 3 \\
\text { Qtr } 4\end{array}$ & $\begin{array}{l}103.9 \\
104.5 \\
105.2 \\
106.8\end{array}$ & $\begin{array}{l}102.8 \\
104.8 \\
105.7 \\
106.1\end{array}$ & $\begin{array}{l}95.1 \\
93.3 \\
93.0 \\
90.9\end{array}$ & $\begin{array}{l}96.6 \\
95.2 \\
95.6 \\
95.9\end{array}$ & $\begin{array}{l}97.0 \\
96.7 \\
97.2 \\
97.7\end{array}$ & $\begin{array}{l}134.2 \\
137.6 \\
137.7 \\
154.7\end{array}$ & $\begin{array}{l}116.0 \\
116.1 \\
117.4 \\
120.5\end{array}$ & $\begin{array}{l}92.9 \\
91.1 \\
91.3 \\
92.1\end{array}$ \\
\hline 2005 & $\begin{array}{l}\text { Qtr } 1 \\
\text { Qtr } 2 \\
\text { Qtr } 3 \\
\text { Qtr } 4\end{array}$ & $\begin{array}{l}108.0 \\
108.9 \\
110.3 \\
110.9\end{array}$ & $\begin{array}{l}106.6 \\
107.5 \\
108.5 \\
107.5\end{array}$ & $\begin{array}{c}96.5 \\
98.7 \\
100.7 \\
101.2\end{array}$ & $\begin{array}{l}95.9 \\
95.7 \\
96.8 \\
96.5\end{array}$ & $\begin{array}{l}98.7 \\
98.8 \\
99.0 \\
99.0\end{array}$ & $\begin{array}{l}161.4 \\
164.4 \\
174.3 \\
187.5\end{array}$ & $\begin{array}{l}121.2 \\
122.4 \\
123.3 \\
124.9\end{array}$ & $\begin{array}{l}92.1 \\
91.5 \\
92.2 \\
92.2\end{array}$ \\
\hline 2006 & $\begin{array}{l}\text { Qtr } 1 \\
\text { Qtr } 2 \\
\text { Qtr } 3 \\
\text { Qtr } 4\end{array}$ & $\begin{array}{l}112.2 \\
114.0 \\
116.0 \\
116.4\end{array}$ & $\begin{array}{l}109.1 \\
109.7 \\
111.0 \\
111.8\end{array}$ & $\begin{array}{l}102.8 \\
104.3 \\
109.4 \\
108.6\end{array}$ & $\begin{array}{l}97.1 \\
96.6 \\
96.6 \\
97.4\end{array}$ & $\begin{array}{l}100.5 \\
100.9 \\
102.3 \\
103.0\end{array}$ & $\begin{array}{l}187.4 \\
199.1 \\
212.9 \\
213.6\end{array}$ & $\begin{array}{l}127.1 \\
129.8 \\
130.7 \\
130.6\end{array}$ & $\begin{array}{l}92.2 \\
93.7 \\
93.7 \\
93.8\end{array}$ \\
\hline 2007 & $\begin{array}{l}\text { Qtr } 1 \\
\text { Qtr } 2 \\
\text { Qtr } 3 \\
\text { Qtr } 4\end{array}$ & $\begin{array}{l}117.0 \\
117.6 \\
118.7 \\
120.4\end{array}$ & $\begin{array}{l}111.5 \\
111.9 \\
112.8 \\
114.5\end{array}$ & $\begin{array}{l}108.6 \\
108.6 \\
108.8 \\
108.3\end{array}$ & $\begin{array}{l}97.5 \\
97.6 \\
97.9 \\
98.9\end{array}$ & $\begin{array}{l}102.9 \\
102.9 \\
103.6 \\
104.3\end{array}$ & $\begin{array}{l}226.2 \\
229.8 \\
234.5 \\
236.7\end{array}$ & $\begin{array}{l}131.3 \\
132.4 \\
134.4 \\
138.9\end{array}$ & $\begin{array}{l}93.8 \\
93.7 \\
94.2 \\
94.5\end{array}$ \\
\hline & & \multicolumn{8}{|c|}{ (Four-quarter percent change) } \\
\hline 2004 & $\begin{array}{l}\text { Qtr } 1 \\
\text { Qtr } 2 \\
\text { Qtr } 3 \\
\text { Qtr } 4\end{array}$ & $\begin{array}{l}1.9 \\
1.8 \\
2.4 \\
3.2\end{array}$ & $\begin{array}{l}3.5 \\
4.3 \\
5.3 \\
5.1\end{array}$ & $\begin{array}{l}-3.9 \\
-4.6 \\
-5.5 \\
-6.7\end{array}$ & $\begin{array}{c}0.7 \\
-0.7 \\
-1.4 \\
-1.1\end{array}$ & $\begin{array}{l}-0.1 \\
-0.3 \\
0.7 \\
1.1\end{array}$ & $\begin{array}{c}7.4 \\
10.2 \\
8.7 \\
15.1\end{array}$ & $\begin{array}{l}3.1 \\
2.5 \\
3.9 \\
6.3\end{array}$ & $\begin{array}{l}-2.7 \\
-4.8 \\
-4.7 \\
-4.2\end{array}$ \\
\hline 2005 & $\begin{array}{l}\text { Qtr } 1 \\
\text { Qtr } 2 \\
\text { Qtr } 3 \\
\text { Qtr } 4\end{array}$ & $\begin{array}{l}3.9 \\
4.2 \\
4.9 \\
3.9\end{array}$ & $\begin{array}{l}3.7 \\
2.6 \\
2.6 \\
1.3\end{array}$ & $\begin{array}{c}1.5 \\
5.7 \\
8.4 \\
11.3\end{array}$ & $\begin{array}{l}-0.7 \\
0.5 \\
1.2 \\
0.6\end{array}$ & $\begin{array}{l}1.8 \\
2.1 \\
1.8 \\
1.3\end{array}$ & $\begin{array}{l}20.2 \\
19.4 \\
26.6 \\
21.2\end{array}$ & $\begin{array}{l}4.5 \\
5.4 \\
5.0 \\
3.6\end{array}$ & $\begin{array}{l}-0.9 \\
0.4 \\
1.0 \\
0.1\end{array}$ \\
\hline 2006 & $\begin{array}{l}\text { Qtr } 1 \\
\text { Qtr } 2 \\
\text { Qtr } 3 \\
\text { Qtr } 4\end{array}$ & $\begin{array}{l}3.9 \\
4.7 \\
5.1 \\
4.9\end{array}$ & $\begin{array}{l}2.4 \\
2.1 \\
2.4 \\
4.0\end{array}$ & $\begin{array}{l}6.5 \\
5.7 \\
8.6 \\
7.3\end{array}$ & $\begin{array}{c}1.2 \\
0.9 \\
-0.2 \\
1.0\end{array}$ & $\begin{array}{l}1.8 \\
2.2 \\
3.3 \\
4.0\end{array}$ & $\begin{array}{l}16.2 \\
21.1 \\
22.2 \\
13.9\end{array}$ & $\begin{array}{l}4.8 \\
6.1 \\
6.0 \\
4.6\end{array}$ & $\begin{array}{l}0.1 \\
2.4 \\
1.6 \\
1.8\end{array}$ \\
\hline 2007 & $\begin{array}{l}\text { Qtr } 1 \\
\text { Qtr } 2 \\
\text { Qtr } 3 \\
\text { Qtr } 4\end{array}$ & $\begin{array}{l}4.3 \\
3.1 \\
2.3 \\
3.5\end{array}$ & $\begin{array}{l}2.2 \\
1.9 \\
1.6 \\
2.4\end{array}$ & $\begin{array}{l}5.6 \\
4.1 \\
-0.6 \\
-0.3\end{array}$ & $\begin{array}{l}0.4 \\
1.0 \\
1.4 \\
1.5\end{array}$ & $\begin{array}{l}2.4 \\
2.0 \\
1.3 \\
1.3\end{array}$ & $\begin{array}{l}20.7 \\
15.4 \\
10.1 \\
10.8\end{array}$ & $\begin{array}{l}3.3 \\
2.0 \\
2.8 \\
6.3\end{array}$ & $\begin{array}{l}1.7 \\
0.0 \\
0.5 \\
0.7\end{array}$ \\
\hline $\begin{array}{l}\text { Memorandum item: } \\
\text { Weight in FSM total }\end{array}$ & & 100.0 & 45.5 & 8.5 & 2.9 & 2.7 & 5.6 & 16.5 & 18.4 \\
\hline
\end{tabular}

Source: Data provided by the FSM authorities. 
Table 6. Federated States of Micronesia: Pohnpei Consumer Price Index, 2001-07

\begin{tabular}{|c|c|c|c|c|c|c|c|c|c|}
\hline & & Total & Food & $\begin{array}{c}\text { Tobacco, } \\
\text { Alcohol, } \\
\text { Betelnut, } \\
\text { Sakau }\end{array}$ & $\begin{array}{l}\text { Clothing, } \\
\text { Footwear }\end{array}$ & Housing & $\begin{array}{l}\text { Fuel, Light \& } \\
\text { Water }\end{array}$ & Services & Miscellaneous \\
\hline & & \multicolumn{8}{|c|}{$(2000=100)$} \\
\hline \multirow[t]{4}{*}{2001} & Qtr 1 & 104.4 & 103.0 & 98.2 & 95.9 & 98.6 & 113.2 & 118.7 & 99.0 \\
\hline & Qtr 2 & 104.4 & 103.0 & 98.1 & 95.9 & 98.8 & 110.4 & 118.6 & 99.9 \\
\hline & Qtr 3 & 102.5 & 101.6 & 96.6 & 96.9 & 98.6 & 110.8 & 120.4 & 92.3 \\
\hline & Qtr 4 & 102.8 & 103.6 & 92.4 & 95.4 & 98.4 & 105.1 & 122.2 & 92.3 \\
\hline \multirow[t]{4}{*}{2002} & Qtr 1 & 103.7 & 105.0 & 90.1 & 97.4 & 100.0 & 112.4 & 121.6 & 92.0 \\
\hline & Qtr 2 & 102.6 & 103.2 & 89.8 & 96.9 & 100.2 & 106.1 & 123.2 & 92.0 \\
\hline & Qtr 3 & 102.6 & 103.1 & 90.9 & 95.0 & 99.4 & 108.1 & 122.1 & 92.1 \\
\hline & Qtr 4 & 102.0 & 101.0 & 92.7 & 94.1 & 99.4 & 110.7 & 122.1 & 92.2 \\
\hline \multirow[t]{4}{*}{2003} & Qtr 1 & 102.2 & 101.0 & 92.7 & 95.7 & 100.1 & 111.3 & 122.2 & 92.4 \\
\hline & Qtr 2 & 103.4 & 103.4 & 92.7 & 96.0 & 100.2 & 111.7 & 122.3 & 92.7 \\
\hline & Qtr 3 & 103.1 & 103.1 & 92.4 & 98.5 & 100.2 & 111.7 & 122.0 & 92.2 \\
\hline & Qtr 4 & 102.8 & 102.8 & 91.8 & 98.3 & 100.2 & 111.7 & 122.1 & 91.5 \\
\hline \multirow[t]{4}{*}{2004} & Qtr 1 & 103.2 & 107.5 & 93.2 & 98.8 & 100.2 & 110.7 & 122.5 & 82.7 \\
\hline & Qtr 2 & 104.2 & 108.2 & 93.2 & 98.8 & 100.1 & 117.6 & 123.4 & 82.8 \\
\hline & Qtr 3 & 104.2 & 108.2 & 93.2 & 98.8 & 100.1 & 117.6 & 123.4 & 82.8 \\
\hline & Qtr 4 & 106.0 & 109.2 & 91.4 & 98.4 & 100.1 & 128.0 & 126.3 & 84.9 \\
\hline \multirow[t]{4}{*}{2005} & Qtr 1 & 107.6 & 111.0 & 96.1 & 98.4 & 100.5 & 132.4 & 127.0 & 84.9 \\
\hline & Qtr 2 & 109.3 & 112.8 & 100.8 & 98.3 & 100.8 & 136.7 & 127.6 & 84.9 \\
\hline & Qtr 3 & 110.9 & 114.5 & 105.5 & 98.2 & 101.2 & 141.0 & 128.2 & 85.0 \\
\hline & Qtr 4 & 111.0 & 114.6 & 105.4 & 97.6 & 101.2 & 141.6 & 128.5 & 85.1 \\
\hline \multirow[t]{4}{*}{2006} & Qtr 1 & 110.9 & 114.3 & 106.3 & 98.1 & 103.2 & 141.3 & 128.0 & 85.0 \\
\hline & Qtr 2 & 112.1 & 114.9 & 108.9 & 96.9 & 104.0 & 143.5 & 131.2 & 85.4 \\
\hline & Qtr 3 & 113.3 & 115.1 & 109.0 & 96.9 & 104.9 & 156.7 & 131.5 & 85.4 \\
\hline & Qtr 4 & 114.6 & 118.0 & 108.5 & 98.7 & 105.1 & 157.6 & 131.4 & 85.5 \\
\hline \multirow[t]{5}{*}{2007} & Qtr 1 & 115.2 & 117.4 & 108.0 & 98.7 & 105.0 & 170.7 & 131.5 & 85.4 \\
\hline & Qtr 2 & 115.2 & 116.2 & 107.6 & 98.7 & 105.0 & 176.4 & 132.6 & 85.4 \\
\hline & Qtr 3 & 116.0 & 117.7 & 107.6 & 99.5 & 105.8 & 176.4 & 133.5 & 85.4 \\
\hline & Qtr 4 & 116.7 & 118.9 & 107.6 & 99.5 & 106.9 & 175.0 & 135.3 & 85.2 \\
\hline & & \multicolumn{8}{|c|}{ (Four-quarter percent change) } \\
\hline \multirow[t]{4}{*}{2004} & Qtr 1 & 1.0 & 6.5 & 0.5 & 3.2 & 0.1 & -0.6 & 0.2 & -10.5 \\
\hline & Qtr 2 & 0.8 & 4.6 & 0.5 & 2.9 & -0.1 & 5.3 & 0.8 & -10.7 \\
\hline & Qtr 3 & 1.0 & 5.0 & 0.8 & 0.3 & -0.1 & 5.3 & 1.1 & -10.2 \\
\hline & Qtr 4 & 3.1 & 6.2 & -0.4 & 0.2 & -0.1 & 14.6 & 3.5 & -7.3 \\
\hline \multirow[t]{4}{*}{2005} & Qtr 1 & 4.3 & 3.2 & 3.1 & -0.4 & 0.3 & 19.6 & 3.7 & 2.6 \\
\hline & Qtr 2 & 4.9 & 4.2 & 8.2 & -0.5 & 0.7 & 16.2 & 3.4 & 2.5 \\
\hline & Qtr 3 & 6.5 & 5.8 & 13.2 & -0.5 & 1.1 & 19.9 & 3.9 & 2.7 \\
\hline & Qtr 4 & 4.7 & 5.0 & 15.3 & -0.9 & 1.1 & 10.6 & 1.7 & 0.3 \\
\hline \multirow[t]{4}{*}{2006} & Qtr 1 & 3.1 & 3.0 & 10.7 & -0.3 & 2.7 & 6.8 & 0.8 & 0.2 \\
\hline & Qtr 2 & 2.6 & 1.9 & 8.0 & -1.4 & 3.2 & 5.0 & 2.9 & 0.6 \\
\hline & Qtr 3 & 2.1 & 0.5 & 3.3 & -1.4 & 3.6 & 11.1 & 2.6 & 0.5 \\
\hline & Qtr 4 & 3.2 & 2.9 & 3.0 & 1.1 & 3.8 & 11.3 & 2.2 & 0.5 \\
\hline \multirow[t]{4}{*}{2007} & Qtr 1 & 3.8 & 2.7 & 1.6 & 0.7 & 1.7 & 20.8 & 2.7 & 0.4 \\
\hline & Qtr 2 & 2.7 & 1.1 & -1.2 & 1.8 & 0.9 & 22.9 & 1.0 & 0.0 \\
\hline & Qtr 3 & 2.4 & 2.2 & -1.3 & 2.7 & 0.9 & 12.6 & 1.5 & 0.0 \\
\hline & Qtr 4 & 1.9 & 0.8 & -0.8 & 0.9 & 1.8 & 11.0 & 3.0 & -0.3 \\
\hline \multicolumn{10}{|l|}{ Memorandum item: } \\
\hline Weight in FSM total & & 37.5 & 16.2 & 3.5 & 1.1 & 1.2 & 2.7 & 5.5 & 7.3 \\
\hline
\end{tabular}

Source: Data provided by the FSM authorities.

CInternational Monetary Fund. Not for Redistribution 
Table 7. Federated States of Micronesia: Chuuk Consumer Price Index, 2001-07

\begin{tabular}{|c|c|c|c|c|c|c|c|c|c|}
\hline & & Total & Food & $\begin{array}{l}\text { Tobacco, } \\
\text { Alcohol, } \\
\text { Betelnut, } \\
\text { Sakau }\end{array}$ & $\begin{array}{l}\text { Clothing, } \\
\text { Footwear }\end{array}$ & Housing & $\begin{array}{l}\text { Fuel, Light \& } \\
\text { Water }\end{array}$ & Services & Miscellaneous \\
\hline & & \multicolumn{8}{|c|}{$(2000=100)$} \\
\hline \multirow[t]{4}{*}{2001} & Qtr 1 & 101.7 & 99.0 & 109.4 & 96.6 & 100.8 & 116.3 & 105.2 & 100.7 \\
\hline & Qtr 2 & 100.9 & 98.0 & 107.7 & 96.4 & 101.2 & 116.3 & 105.3 & 99.5 \\
\hline & Qtr 3 & 102.3 & 99.5 & 113.6 & 95.9 & 100.0 & 121.8 & 106.4 & 98.2 \\
\hline & Qtr 4 & 102.9 & 98.9 & 111.4 & 96.1 & 99.8 & 149.2 & 106.1 & 97.2 \\
\hline \multirow[t]{4}{*}{2002} & Qtr 1 & 102.6 & 99.8 & 100.7 & 97.1 & 101.7 & 150.6 & 105.1 & 97.6 \\
\hline & Qtr 2 & 103.4 & 100.4 & 108.6 & 96.2 & 99.7 & 150.6 & 105.2 & 97.3 \\
\hline & Qtr 3 & 103.0 & 99.9 & 108.6 & 96.2 & 99.6 & 147.8 & 105.1 & 97.6 \\
\hline & Qtr 4 & 103.3 & 99.6 & 109.4 & 96.4 & 101.8 & 153.3 & 105.1 & 97.7 \\
\hline \multirow[t]{4}{*}{2003} & Qtr 1 & 102.7 & 98.7 & 105.9 & 96.0 & 94.3 & 156.5 & 105.9 & 97.9 \\
\hline & Qtr 2 & 103.3 & 99.5 & 101.8 & 96.0 & 94.0 & 159.3 & 107.6 & 98.4 \\
\hline & Qtr 3 & 103.5 & 99.2 & 103.5 & 96.2 & 92.5 & 164.8 & 107.4 & 98.8 \\
\hline & Qtr 4 & 105.6 & 100.7 & 103.5 & 96.4 & 93.5 & 187.6 & 107.7 & 100.3 \\
\hline \multirow[t]{4}{*}{2004} & Qtr 1 & 106.3 & 101.1 & 95.2 & 95.3 & 93.9 & 188.6 & 113.7 & 100.6 \\
\hline & Qtr 2 & 106.9 & 104.1 & 88.6 & 95.4 & 92.6 & 188.6 & 114.8 & 96.2 \\
\hline & Qtr 3 & 108.3 & 106.0 & 88.8 & 94.8 & 92.9 & 188.6 & 116.5 & 96.7 \\
\hline & Qtr 4 & 109.6 & 105.7 & 87.8 & 94.8 & 93.3 & 212.4 & 119.3 & 97.1 \\
\hline \multirow[t]{4}{*}{2005} & Qtr 1 & 109.8 & 105.2 & 89.4 & 94.9 & 93.9 & 214.7 & 120.3 & 97.1 \\
\hline & Qtr 2 & 109.9 & 105.7 & 89.4 & 94.4 & 93.9 & 214.7 & 120.3 & 96.4 \\
\hline & Qtr 3 & 111.2 & 106.0 & 88.3 & 95.1 & 93.4 & 235.8 & 120.7 & 97.6 \\
\hline & Qtr 4 & 112.2 & 104.0 & 89.0 & 95.0 & 93.4 & 272.5 & 123.0 & 97.6 \\
\hline \multirow[t]{4}{*}{2006} & Qtr 1 & 115.3 & 107.5 & 92.5 & 95.3 & 94.1 & 272.5 & 129.1 & 97.8 \\
\hline & Qtr 2 & 117.1 & 107.6 & 92.8 & 95.3 & 94.4 & 280.3 & 133.4 & 102.0 \\
\hline & Qtr 3 & 119.9 & 109.6 & 107.9 & 95.3 & 95.2 & 290.0 & 135.1 & 102.0 \\
\hline & Qtr 4 & 119.8 & 108.7 & 108.9 & 95.7 & 96.2 & 296.4 & 135.0 & 102.1 \\
\hline \multirow[t]{5}{*}{2007} & Qtr 1 & 120.5 & 108.2 & 107.8 & 95.5 & 96.1 & 312.8 & 136.8 & 102.1 \\
\hline & Qtr 2 & 121.4 & 109.3 & 107.8 & 95.7 & 96.1 & 314.7 & 138.4 & 102.0 \\
\hline & Qtr 3 & 122.1 & 109.8 & 107.8 & 95.7 & 96.1 & 317.0 & 140.1 & 102.1 \\
\hline & Qtr 4 & 124.7 & 111.7 & 107.8 & 98.4 & 96.0 & 317.0 & 148.8 & 103.3 \\
\hline & & \multicolumn{8}{|c|}{ (Four-quarter percent change) } \\
\hline \multirow[t]{4}{*}{2004} & Qtr 1 & 3.5 & 2.4 & -10.1 & -0.8 & -0.4 & 20.5 & 7.4 & 2.8 \\
\hline & Qtr 2 & 3.5 & 4.6 & -13.0 & -0.6 & -1.5 & 18.4 & 6.7 & -2.2 \\
\hline & Qtr 3 & 4.6 & 6.8 & -14.2 & -1.4 & 0.4 & 14.4 & 8.4 & -2.1 \\
\hline & Qtr 4 & 3.8 & 4.9 & -15.2 & -1.6 & -0.2 & 13.2 & 10.8 & -3.2 \\
\hline \multirow[t]{4}{*}{2005} & Qtr 1 & 3.3 & 4.1 & -6.1 & -0.4 & 0.0 & 13.9 & 5.8 & -3.5 \\
\hline & Qtr 2 & 2.8 & 1.5 & 0.9 & -0.9 & 1.4 & 13.9 & 4.8 & 0.2 \\
\hline & Qtr 3 & 2.7 & 0.0 & -0.6 & 0.3 & 0.5 & 25.1 & 3.6 & 0.9 \\
\hline & Qtr 4 & 2.4 & -1.5 & 1.5 & 0.2 & 0.0 & 28.3 & 3.1 & 0.6 \\
\hline \multirow[t]{4}{*}{2006} & Qtr 1 & 5.0 & 2.1 & 3.5 & 0.4 & 0.2 & 26.9 & 7.3 & 0.7 \\
\hline & Qtr 2 & 6.5 & 1.8 & 3.8 & 0.9 & 0.5 & 30.6 & 10.9 & 5.8 \\
\hline & Qtr 3 & 7.8 & 3.4 & 22.2 & 0.2 & 2.0 & 23.0 & 11.9 & 4.5 \\
\hline & Qtr 4 & 6.7 & 4.4 & 22.3 & 0.7 & 3.0 & 8.8 & 9.7 & 4.6 \\
\hline \multirow[t]{4}{*}{2007} & Qtr 1 & 4.5 & 0.7 & 16.5 & 0.2 & 2.1 & 14.8 & 6.0 & 4.4 \\
\hline & Qtr 2 & 3.7 & 1.5 & 16.2 & 0.4 & 1.8 & 12.2 & 3.7 & 0.1 \\
\hline & Qtr 3 & 1.8 & 0.2 & -0.1 & 0.4 & 1.0 & 9.3 & 3.7 & 0.1 \\
\hline & Qtr 4 & 4.1 & 2.8 & -1.0 & 2.9 & -0.1 & 7.0 & 10.2 & 1.1 \\
\hline \multicolumn{10}{|l|}{ Memorandum item: } \\
\hline Weight in FSM total & & 41.6 & 22.0 & 2.8 & 1.1 & 0.9 & 1.9 & 6.7 & 6.1 \\
\hline
\end{tabular}

Source: Data provided by the FSM authorities. 
Table 8. Federated States of Micronesia: Kosrae Consumer Price Index, 2001-07

\begin{tabular}{|c|c|c|c|c|c|c|c|c|c|}
\hline & & Total & Food & $\begin{array}{c}\text { Tobacco, } \\
\text { Alcohol, } \\
\text { Betelnut, } \\
\text { Sakau }\end{array}$ & $\begin{array}{l}\text { Clothing, } \\
\text { Footwear }\end{array}$ & Housing & $\begin{array}{l}\text { Fuel, Light \& } \\
\text { Water }\end{array}$ & Services & Miscellaneous \\
\hline & & \multicolumn{8}{|c|}{$(2000=100)$} \\
\hline \multirow[t]{4}{*}{2001} & Qtr 1 & 101.8 & 99.9 & 102.0 & 102.0 & 102.3 & 99.5 & 103.6 & 103.6 \\
\hline & Qtr 2 & 101.3 & 99.6 & 102.0 & 102.2 & 102.8 & 99.5 & 104.0 & 101.9 \\
\hline & Qtr 3 & 100.7 & 98.8 & 104.5 & 101.8 & 95.3 & 99.2 & 103.6 & 101.0 \\
\hline & Qtr 4 & 100.4 & 99.4 & 95.4 & 102.8 & 97.5 & 99.2 & 103.6 & 100.0 \\
\hline \multirow[t]{4}{*}{2002} & Qtr 1 & 99.1 & 98.1 & 95.0 & 100.0 & 94.6 & 99.4 & 101.5 & 99.2 \\
\hline & Qtr 2 & 97.2 & 93.2 & 90.4 & 99.9 & 92.7 & 99.5 & 101.8 & 100.5 \\
\hline & Qtr 3 & 96.7 & 92.9 & 90.4 & 99.9 & 93.5 & 99.5 & 101.8 & 98.9 \\
\hline & Qtr 4 & 96.6 & 92.5 & 90.4 & 99.9 & 93.5 & 99.5 & 101.8 & 99.0 \\
\hline \multirow[t]{4}{*}{2003} & Qtr 1 & 96.5 & 92.2 & 90.4 & 100.2 & 93.5 & 99.5 & 101.8 & 98.9 \\
\hline & Qtr 2 & 96.4 & 92.3 & 90.4 & 100.1 & 93.6 & 99.5 & 101.8 & 98.4 \\
\hline & Qtr 3 & 96.7 & 93.0 & 92.8 & 100.2 & 93.6 & 99.5 & 101.8 & 98.1 \\
\hline & Qtr 4 & 96.6 & 92.8 & 92.8 & 99.9 & 93.6 & 99.5 & 101.7 & 98.3 \\
\hline \multirow[t]{4}{*}{2004} & Qtr 1 & 98.0 & 94.2 & 93.9 & 99.5 & 92.8 & 99.5 & 102.3 & 101.3 \\
\hline & Qtr 2 & 100.9 & 99.4 & 95.1 & 100.7 & 93.8 & 101.1 & 105.1 & 101.2 \\
\hline & Qtr 3 & 101.0 & 98.5 & 95.1 & 100.7 & 93.8 & 101.1 & 107.1 & 101.3 \\
\hline & Qtr 4 & 106.0 & 100.8 & 98.0 & 104.0 & 93.9 & 188.0 & 110.5 & 99.8 \\
\hline \multirow[t]{4}{*}{2005} & Qtr 1 & 106.8 & 101.4 & 106.8 & 104.0 & 93.9 & 184.0 & 112.1 & 100.1 \\
\hline & Qtr 2 & 108.2 & 101.5 & 106.8 & 104.0 & 93.9 & 197.9 & 115.9 & 100.1 \\
\hline & Qtr 3 & 109.6 & 101.7 & 106.8 & 104.0 & 93.9 & 211.8 & 119.7 & 100.1 \\
\hline & Qtr 4 & 111.0 & 101.9 & 106.8 & 104.0 & 93.9 & 225.7 & 123.6 & 100.1 \\
\hline \multirow[t]{4}{*}{2006} & Qtr 1 & 110.7 & 101.3 & 106.8 & 104.6 & 95.6 & 227.1 & 122.6 & 100.0 \\
\hline & Qtr 2 & 111.8 & 104.7 & 106.8 & 103.7 & 96.6 & 227.1 & 121.6 & 99.7 \\
\hline & Qtr 3 & 112.6 & 107.2 & 106.8 & 103.7 & 96.6 & 231.8 & 121.4 & 98.4 \\
\hline & Qtr 4 & 112.6 & 108.2 & 106.8 & 104.2 & 96.6 & 231.8 & 118.7 & 99.1 \\
\hline \multirow{5}{*}{2007} & Qtr 1 & 112.8 & 108.8 & 108.0 & 104.3 & 96.6 & 233.1 & 117.7 & 99.3 \\
\hline & Qtr 2 & 113.4 & 109.5 & 108.4 & 104.5 & 96.9 & 236.8 & 118.1 & 99.3 \\
\hline & Qtr 3 & 116.8 & 111.7 & 115.4 & 105.0 & 99.5 & 236.8 & 128.8 & 98.9 \\
\hline & Qtr 4 & 119.2 & 113.4 & 117.5 & 104.8 & 101.2 & 258.7 & 130.1 & 101.0 \\
\hline & & \multicolumn{8}{|c|}{ (Four-quarter percent change) } \\
\hline \multirow[t]{4}{*}{2003} & Qtr 1 & -2.6 & -6.1 & -4.8 & 0.1 & -1.2 & 0.2 & 0.3 & -0.2 \\
\hline & Qtr 2 & -0.9 & -1.0 & 0.0 & 0.3 & 0.9 & 0.0 & 0.0 & -2.0 \\
\hline & Qtr 3 & 0.0 & 0.2 & 2.6 & 0.3 & 0.2 & 0.0 & 0.0 & -0.8 \\
\hline & Qtr 4 & 0.0 & 0.4 & 2.6 & 0.0 & 0.2 & 0.0 & -0.1 & -0.7 \\
\hline \multirow[t]{4}{*}{2004} & Qtr 1 & 1.6 & 2.2 & 3.9 & -0.6 & -0.7 & 0.0 & 0.4 & 2.4 \\
\hline & Qtr 2 & 4.7 & 7.7 & 5.2 & 0.6 & 0.3 & 1.6 & 3.3 & 2.8 \\
\hline & Qtr 3 & 4.4 & 5.9 & 2.5 & 0.6 & 0.3 & 1.6 & 5.3 & 3.3 \\
\hline & Qtr 4 & 9.6 & 8.6 & 5.6 & 4.2 & 0.3 & 88.9 & 8.6 & 1.5 \\
\hline \multirow[t]{4}{*}{2005} & Qtr 1 & 8.9 & 7.6 & 13.7 & 4.5 & 1.1 & 84.9 & 9.6 & -1.2 \\
\hline & Qtr 2 & 7.2 & 2.1 & 12.3 & 3.3 & 0.0 & 95.8 & 10.3 & -1.1 \\
\hline & Qtr 3 & 8.5 & 3.3 & 12.3 & 3.3 & 0.0 & 109.5 & 11.8 & -1.2 \\
\hline & Qtr 4 & 4.8 & 1.1 & 9.0 & 0.0 & 0.0 & 20.1 & 11.8 & 0.3 \\
\hline \multirow[t]{4}{*}{2006} & Qtr 1 & 3.7 & -0.1 & 0.0 & 0.6 & 1.8 & 23.4 & 9.4 & 0.0 \\
\hline & Qtr 2 & 3.3 & 3.1 & 0.0 & -0.3 & 2.9 & 14.7 & 4.9 & -0.3 \\
\hline & Qtr 3 & 2.8 & 5.4 & 0.0 & -0.3 & 2.9 & 9.4 & 1.4 & -1.6 \\
\hline & Qtr 4 & 1.4 & 6.2 & 0.0 & 0.1 & 2.9 & 2.7 & -3.9 & -0.9 \\
\hline \multirow[t]{4}{*}{2007} & Qtr 1 & 1.9 & 7.4 & 1.1 & -0.4 & 1.1 & 2.7 & -4.0 & -0.8 \\
\hline & Qtr 2 & 1.4 & 4.5 & 1.5 & 0.8 & 0.3 & 4.3 & -2.9 & -0.4 \\
\hline & Qtr 3 & 3.7 & 4.2 & 8.1 & 1.3 & 3.0 & 2.2 & 6.1 & 0.5 \\
\hline & Qtr 4 & 5.8 & 4.8 & 10.1 & 0.6 & 4.7 & 11.6 & 9.6 & 1.9 \\
\hline \multicolumn{10}{|l|}{ Memorandum item: } \\
\hline Weight in FSM total & & 6.0 & 2.5 & 0.2 & 0.4 & 0.1 & 0.2 & 1.3 & 1.4 \\
\hline
\end{tabular}

Source: Data provided by the FSM authorities. 
Table 9. Federated States of Micronesia: Yap Consumer Price Index, 2001-07

\begin{tabular}{|c|c|c|c|c|c|c|c|c|c|}
\hline & & Total & Food & $\begin{array}{c}\text { Tobacco, } \\
\text { Alcohol, } \\
\text { Betelnut, } \\
\text { Sakau }\end{array}$ & $\begin{array}{l}\text { Clothing, } \\
\text { Footwear }\end{array}$ & Housing & $\begin{array}{c}\text { Fuel, Light \& } \\
\text { Water }\end{array}$ & Services & Miscellaneous \\
\hline & & \multicolumn{8}{|c|}{$(2000=100)$} \\
\hline \multirow[t]{4}{*}{2001} & Qtr 1 & 102.5 & 100.7 & 104.5 & 94.4 & 99.2 & 105.5 & 110.1 & 98.0 \\
\hline & Qtr 2 & 103.4 & 101.9 & 108.4 & 93.3 & 100.5 & 105.5 & 110.4 & 97.5 \\
\hline & Qtr 3 & 103.6 & 101.5 & 109.4 & 92.5 & 97.1 & 105.2 & 112.1 & 97.6 \\
\hline & Qtr 4 & 103.0 & 101.9 & 105.6 & 91.8 & 96.5 & 102.4 & 112.8 & 96.9 \\
\hline \multirow[t]{4}{*}{2002} & Qtr 1 & 102.7 & 101.0 & 105.1 & 91.6 & 96.3 & 102.6 & 113.1 & 97.0 \\
\hline & Qtr 2 & 103.4 & 101.8 & 105.9 & 91.8 & 96.5 & 104.8 & 113.6 & 97.1 \\
\hline & Qtr 3 & 102.5 & 100.2 & 104.0 & 91.5 & 96.0 & 104.9 & 113.6 & 96.6 \\
\hline & Qtr 4 & 102.7 & 100.7 & 104.7 & 91.4 & 95.7 & 104.9 & 113.9 & 96.4 \\
\hline \multirow[t]{4}{*}{2003} & Qtr 1 & 102.1 & 100.2 & 100.9 & 91.1 & 96.2 & 104.9 & 114.1 & 96.2 \\
\hline & Qtr 2 & 101.5 & 99.0 & 102.3 & 90.6 & 95.9 & 96.1 & 114.9 & 96.3 \\
\hline & Qtr 3 & 102.0 & 100.1 & 102.3 & 91.1 & 96.2 & 96.0 & 114.2 & 97.2 \\
\hline & Qtr 4 & 101.9 & 99.9 & 99.5 & 91.0 & 95.1 & 96.2 & 115.4 & 97.5 \\
\hline \multirow[t]{4}{*}{2004} & Qtr 1 & 101.5 & 99.5 & 98.3 & 90.5 & 96.2 & 96.5 & 115.2 & 97.4 \\
\hline & Qtr 2 & 100.1 & 99.1 & 100.1 & 76.0 & 97.0 & 96.6 & 110.7 & 95.7 \\
\hline & Qtr 3 & 100.7 & 99.8 & 98.2 & 81.7 & 99.2 & 97.0 & 113.1 & 95.4 \\
\hline & Qtr 4 & 101.3 & 100.3 & 93.8 & 81.8 & 101.0 & 99.8 & 117.0 & 95.5 \\
\hline \multirow[t]{4}{*}{2005} & Qtr 1 & 104.5 & 100.5 & 106.2 & 81.7 & 104.5 & 127.6 & 116.8 & 95.2 \\
\hline & Qtr 2 & 105.3 & 101.3 & 107.2 & 81.8 & 103.8 & 130.0 & 120.2 & 93.6 \\
\hline & Qtr 3 & 106.8 & 102.9 & 109.5 & 89.5 & 105.2 & 131.3 & 121.6 & 94.4 \\
\hline & Qtr 4 & 107.0 & 101.9 & 110.6 & 89.5 & 105.3 & 132.1 & 123.1 & 94.4 \\
\hline \multirow[t]{4}{*}{2006} & Qtr 1 & 107.5 & 103.4 & 110.7 & 91.4 & 106.7 & 132.1 & 122.9 & 94.3 \\
\hline & Qtr 2 & 111.0 & 104.6 & 112.1 & 91.8 & 106.5 & 187.5 & 122.6 & 94.2 \\
\hline & Qtr 3 & 113.2 & 105.6 & 112.6 & 91.8 & 110.1 & 215.9 & 123.3 & 94.4 \\
\hline & Qtr 4 & 112.8 & 107.2 & 108.5 & 91.8 & 111.9 & 202.9 & 124.4 & 94.5 \\
\hline \multirow[t]{5}{*}{2007} & Qtr 1 & 113.6 & 107.9 & 110.8 & 92.6 & 111.8 & 208.2 & 124.3 & 94.6 \\
\hline & Qtr 2 & 114.5 & 110.4 & 111.4 & 92.6 & 111.7 & 208.5 & 124.6 & 94.4 \\
\hline & Qtr 3 & 116.9 & 110.5 & 111.6 & 92.1 & 112.9 & 236.0 & 125.8 & 96.7 \\
\hline & Qtr 4 & 118.3 & 113.1 & 109.1 & 91.3 & 114.1 & 250.0 & 126.8 & 96.0 \\
\hline & & \multicolumn{8}{|c|}{ (Four-quarter percent change) } \\
\hline \multirow[t]{4}{*}{2004} & Qtr 1 & -0.5 & -0.7 & -2.6 & -0.7 & 0.1 & -8.0 & 1.0 & 1.2 \\
\hline & Qtr 2 & -1.4 & 0.2 & -2.1 & -16.1 & 1.1 & 0.5 & -3.6 & -0.6 \\
\hline & Qtr 3 & -1.3 & -0.3 & -3.9 & -10.3 & 3.1 & 1.0 & -0.9 & -1.8 \\
\hline & Qtr 4 & -0.6 & 0.4 & -5.7 & -10.1 & 6.2 & 3.7 & 1.4 & -2.0 \\
\hline \multirow[t]{4}{*}{2005} & Qtr 1 & 2.9 & 1.1 & 8.0 & -9.7 & 8.6 & 32.2 & 1.4 & -2.3 \\
\hline & Qtr 2 & 5.2 & 2.2 & 7.1 & 7.6 & 7.0 & 34.6 & 8.6 & -2.3 \\
\hline & Qtr 3 & 6.1 & 3.1 & 11.4 & 9.5 & 6.1 & 35.4 & 7.5 & -1.1 \\
\hline & Qtr 4 & 5.6 & 1.6 & 17.9 & 9.4 & 4.2 & 32.4 & 5.2 & -1.1 \\
\hline \multirow[t]{4}{*}{2006} & Qtr 1 & 2.9 & 2.8 & 4.3 & 11.9 & 2.1 & 3.6 & 5.2 & -0.9 \\
\hline & Qtr 2 & 5.5 & 3.2 & 4.6 & 12.2 & 2.6 & 44.2 & 2.0 & 0.7 \\
\hline & Qtr 3 & 6.0 & 2.6 & 2.9 & 2.7 & 4.6 & 64.4 & 1.4 & 0.1 \\
\hline & Qtr 4 & 5.4 & 5.2 & -1.9 & 2.6 & 6.3 & 53.6 & 1.1 & 0.1 \\
\hline \multirow[t]{4}{*}{2007} & Qtr 1 & 5.7 & 4.4 & 0.1 & 1.3 & 4.8 & 57.6 & 1.1 & 0.3 \\
\hline & Qtr 2 & 3.2 & 5.6 & -0.6 & 0.8 & 4.9 & 11.2 & 1.6 & 0.2 \\
\hline & Qtr 3 & 3.2 & 4.7 & -0.9 & 0.3 & 2.5 & 9.3 & 2.0 & 2.4 \\
\hline & Qtr 4 & 4.8 & 5.5 & 0.6 & -0.6 & 2.1 & 23.2 & 1.9 & 1.6 \\
\hline \multicolumn{10}{|l|}{ Memorandum item: } \\
\hline Weight in FSM total & & 14.9 & 4.8 & 1.9 & 0.3 & 0.5 & 0.8 & 3.0 & 3.5 \\
\hline
\end{tabular}

Source: Data provided by the FSM authorities.

CInternational Monetary Fund. Not for Redistribution 
Table 10. Federated States of Micronesia: Consolidated General Government Finances, FY2002-07 1/

\begin{tabular}{|c|c|c|c|c|c|c|}
\hline & FY2002 & FY2003 & FY2004 & FY2005 & $\begin{array}{r}\text { FY2006 } \\
\text { Est. }\end{array}$ & $\begin{array}{r}\text { FY2007 } \\
\text { Est. }\end{array}$ \\
\hline & \multicolumn{6}{|c|}{ (In millions of U.S. dollars) } \\
\hline Total revenue and grants & 160.3 & 170.4 & 133.6 & 135.9 & 139.7 & 145.2 \\
\hline Total revenue & 47.0 & 48.4 & 59.8 & 52.1 & 54.9 & 52.8 \\
\hline Tax revenue & 26.3 & 24.1 & 27.3 & 29.2 & 29.7 & 27.8 \\
\hline Wages and salary tax & 7.5 & 6.5 & 7.3 & 6.5 & 6.7 & 5.4 \\
\hline Gross revenue tax & 6.2 & 5.1 & 6.8 & 6.7 & 5.7 & 6.2 \\
\hline Import tax:Fuel & 0.8 & 0.6 & 0.7 & 0.6 & 0.7 & 0.8 \\
\hline Import tax:All others & 5.9 & 5.4 & 6.2 & 8.0 & 7.8 & 7.3 \\
\hline Cigarette tax & 0.0 & 0.0 & 0.0 & 0.0 & 2.3 & 1.5 \\
\hline All other tax (National) & 0.9 & 0.2 & 0.0 & 0.0 & 0.0 & 0.0 \\
\hline State tax revenue & 4.9 & 6.2 & 6.3 & 7.4 & 6.5 & 6.8 \\
\hline Nontax revenue & 20.7 & 24.3 & 32.5 & 22.9 & 25.2 & 24.9 \\
\hline Fishing access revenue & 10.6 & 11.8 & 12.1 & 13.3 & 13.2 & 15.0 \\
\hline Dividend and interest income & 2.3 & 1.3 & 3.3 & 2.0 & 1.4 & 2.7 \\
\hline Other nontax revenues & 7.9 & 11.2 & 17.1 & 7.6 & 10.6 & 7.2 \\
\hline Grants & 113.3 & 122.1 & 73.9 & 83.9 & 84.8 & 92.5 \\
\hline Grants from abroad & 113.3 & 122.1 & 73.9 & 83.9 & 84.8 & 92.5 \\
\hline Current grants & 81.5 & 90.2 & 73.9 & 83.9 & 81.8 & 85.6 \\
\hline Compact General & 47.7 & 47.7 & 0.0 & 0.0 & 0.0 & 0.0 \\
\hline Compact Special & 18.2 & 18.7 & 52.1 & 56.0 & 59.3 & 60.6 \\
\hline Other:Current & 15.5 & 23.8 & 21.8 & 27.9 & 22.5 & 25.0 \\
\hline Capital grants & 31.8 & 31.8 & 0.0 & 0.0 & 3.0 & 6.9 \\
\hline Total expenditure & 143.0 & 164.1 & 169.5 & 148.2 & 155.0 & 153.0 \\
\hline Current expenditure & 119.7 & 138.3 & 126.8 & 136.8 & 148.4 & 139.9 \\
\hline Expenditure on goods and services & 113.1 & 131.3 & 120.5 & 131.4 & 142.8 & 136.8 \\
\hline Wages and salaries & 58.1 & 46.1 & 27.8 & 29.3 & 31.1 & 30.1 \\
\hline Travel & 7.8 & 2.9 & 2.3 & 2.3 & 2.8 & 2.6 \\
\hline Other & 47.2 & 82.3 & 90.4 & 99.8 & 108.9 & 104.1 \\
\hline Subsidies & 4.4 & 3.5 & 2.4 & 1.3 & 1.2 & 2.4 \\
\hline Transfers & 2.2 & 3.4 & 4.0 & 4.1 & 4.4 & 0.7 \\
\hline Capital expenditure & 23.3 & 25.7 & 42.7 & 11.4 & 6.6 & 13.1 \\
\hline Acquisition of fixed capital & 4.6 & 11.6 & 2.8 & 5.9 & 2.7 & 2.7 \\
\hline Multi-purpose development projects & 17.7 & 14.1 & 11.6 & 5.6 & 3.8 & 10.4 \\
\hline Capital Transfers & 1.0 & 0.0 & 28.3 & 0.0 & 0.0 & 0.0 \\
\hline Overall balance & 17.3 & 6.4 & -35.9 & -12.3 & -15.3 & -7.8 \\
\hline Current balance & 8.8 & 0.3 & 6.8 & -0.9 & -11.7 & -1.5 \\
\hline Capital balance & 8.5 & 6.1 & -42.7 & -11.4 & -3.6 & -6.2 \\
\hline
\end{tabular}

Source: Data provided by the FSM authorities; and Fund staff estimates.

1/ Government Financial Statistics (GFS) basis. Fiscal year ending September 30. 
Table 11. Federated States of Micronesia: National Government Finances, FY2002-07 1/

\begin{tabular}{|c|c|c|c|c|c|c|}
\hline & FY2002 & FY2003 & FY2004 & FY2005 & $\begin{array}{r}\text { FY2006 } \\
\text { Est. }\end{array}$ & $\begin{array}{r}\text { FY2007 } \\
\text { Est. }\end{array}$ \\
\hline & \multicolumn{6}{|c|}{ (In millions of U.S. dollars) } \\
\hline Total revenue and grants & 42.8 & 51.6 & 49.2 & 46.8 & 45.6 & 55.1 \\
\hline Total revenue & 22.0 & 23.7 & 30.5 & 28.8 & 27.3 & 29.1 \\
\hline Tax revenue & 7.5 & 6.3 & 9.5 & 11.1 & 11.0 & 10.4 \\
\hline Wages and salary tax & 2.8 & 2.3 & 3.1 & 3.3 & 3.2 & 2.4 \\
\hline Gross revenue tax & 1.9 & 1.7 & 3.5 & 3.5 & 2.4 & 3.1 \\
\hline Import tax: fuel & 0.1 & 0.1 & 0.2 & 0.1 & 0.1 & 0.1 \\
\hline Import tax: all others & 1.8 & 1.9 & 2.8 & 4.1 & 4.3 & 4.0 \\
\hline Cigarette tax & 0.0 & 0.0 & 0.0 & 0.0 & 1.0 & 0.7 \\
\hline All other tax (national) & 0.9 & 0.2 & 0.0 & 0.0 & 0.0 & 0.0 \\
\hline Nontax revenue & 14.5 & 17.4 & 21.0 & 17.7 & 16.3 & 18.7 \\
\hline Fishing access revenue & 10.6 & 11.8 & 12.1 & 13.3 & 13.2 & 15.0 \\
\hline Dividend and interest income & 0.3 & 0.5 & 2.5 & 0.4 & 0.3 & 0.6 \\
\hline Other nontax revenues & 3.6 & 5.0 & 6.4 & 4.0 & 2.8 & 3.1 \\
\hline Grants & 20.8 & 27.9 & 18.7 & 18.0 & 18.3 & 26.0 \\
\hline Grants from abroad & 20.8 & 27.9 & 18.7 & 18.0 & 18.3 & 26.0 \\
\hline Current grants & 17.8 & 25.0 & 18.7 & 18.0 & 15.4 & 19.2 \\
\hline Compact general & 7.0 & 7.0 & 0.0 & 0.0 & 0.0 & 0.0 \\
\hline Compact special & 4.8 & 4.8 & 9.1 & 6.4 & 5.4 & 3.3 \\
\hline Other:Current & 6.1 & 13.2 & 9.6 & 11.5 & 10.1 & 15.8 \\
\hline Capital grants & 3.0 & 3.0 & 0.0 & 0.0 & 2.9 & 6.9 \\
\hline Total expenditure and net lending & 44.4 & 55.6 & 55.7 & 48.4 & 50.7 & 56.7 \\
\hline Total expenditure & 44.4 & 55.6 & 55.7 & 48.4 & 50.7 & 56.7 \\
\hline Current expenditure & 40.8 & 53.4 & 45.6 & 46.7 & 48.1 & 48.0 \\
\hline Expenditure on goods and services & 35.2 & 48.1 & 40.7 & 41.9 & 43.5 & 46.7 \\
\hline Wages and salaries & 12.7 & 0.0 & 0.0 & 0.0 & 0.0 & 0.0 \\
\hline Travel & 4.4 & 0.0 & 0.0 & 0.0 & 0.0 & 0.0 \\
\hline Other & 18.0 & 48.1 & 40.7 & 41.9 & 43.5 & 46.7 \\
\hline Subsidies & 2.2 & 1.7 & 1.0 & 0.6 & 0.6 & 0.8 \\
\hline Transfers & 3.4 & 3.7 & 4.0 & 4.2 & 4.1 & 0.5 \\
\hline Including transfers to state governments & 2.3 & 0.9 & 0.4 & 0.7 & 0.4 & 0.4 \\
\hline Capital expenditure & 3.7 & 2.2 & 10.1 & 1.7 & 2.6 & 8.7 \\
\hline Acquisition of fixed capital & 1.4 & 0.0 & 0.0 & 0.0 & 0.0 & 0.0 \\
\hline Multi-purpose development projects & 1.3 & 2.2 & 2.1 & 1.7 & 2.6 & 8.7 \\
\hline Capital transfers & 1.0 & 0.0 & 8.0 & 0.0 & 0.0 & 0.0 \\
\hline Overall balance & -1.6 & -4.0 & -6.6 & -1.6 & -6.1 & -2.3 \\
\hline Current balance & -0.9 & -4.8 & 3.5 & 0.1 & -6.4 & -0.5 \\
\hline Capital balance & -0.7 & 0.8 & -10.1 & -1.7 & 0.3 & -1.8 \\
\hline
\end{tabular}

Source: Data provided by the FSM authorities; and Fund staff estimates.

1/ Government Financial Statistics (GFS) basis. Fiscal year ending September 30. 
Table 12. Federated States of Micronesia: Pohnpei Government Finances, FY2002-07 1/

\begin{tabular}{|c|c|c|c|c|c|c|}
\hline & FY2002 & FY2003 & FY2004 & FY2005 & $\begin{array}{r}\text { FY2006 } \\
\text { Est. }\end{array}$ & $\begin{array}{r}\text { FY2007 } \\
\text { Est. }\end{array}$ \\
\hline & \multicolumn{6}{|c|}{ (In millions of U.S. dollars) } \\
\hline Total revenue and grants & 38.1 & 38.5 & 34.8 & 29.9 & 31.1 & 31.1 \\
\hline Total revenue & 10.8 & 11.2 & 14.7 & 10.3 & 12.8 & 11.1 \\
\hline Tax revenue & 9.8 & 8.0 & 7.5 & 8.2 & 8.5 & 8.2 \\
\hline Wages and salary tax & 2.7 & 2.1 & 1.8 & 1.5 & 1.7 & 1.5 \\
\hline Gross revenue tax & 2.2 & 1.8 & 1.4 & 1.4 & 1.7 & 1.6 \\
\hline Import tax: fuel & 0.3 & 0.2 & 0.2 & 0.1 & 0.3 & 0.3 \\
\hline Import tax: all others & 2.0 & 1.5 & 1.3 & 1.5 & 1.4 & 1.4 \\
\hline Cigarette tax & 0.0 & 0.0 & 0.0 & 0.0 & 0.7 & 0.5 \\
\hline State tax revenue & 2.4 & 2.4 & 2.7 & 3.5 & 2.8 & 2.8 \\
\hline Nontax revenue & 1.1 & 3.2 & 7.2 & 2.2 & 4.3 & 2.9 \\
\hline Dividend and interest income & 0.3 & 0.3 & 0.4 & 1.2 & 0.8 & 1.7 \\
\hline Other nontax revenues & 0.7 & 2.9 & 6.8 & 1.0 & 3.5 & 1.1 \\
\hline Grants & 27.3 & 27.3 & 20.1 & 19.6 & 18.3 & 20.0 \\
\hline Grants from abroad & 26.5 & 27.1 & 19.9 & 19.5 & 18.2 & 19.9 \\
\hline Current grants & 18.4 & 19.0 & 19.9 & 19.5 & 18.2 & 19.9 \\
\hline Compact general & 11.2 & 11.2 & 0.0 & 0.0 & 0.0 & 0.0 \\
\hline Compact special & 3.7 & 3.9 & 15.4 & 16.0 & 16.6 & 17.3 \\
\hline Other:Current & 3.4 & 3.8 & 4.5 & 3.6 & 1.6 & 2.6 \\
\hline Capital grants & 8.1 & 8.1 & 0.0 & 0.0 & 0.0 & 0.0 \\
\hline Grants from national government & 0.8 & 0.2 & 0.2 & 0.1 & 0.1 & 0.1 \\
\hline Current & 0.8 & 0.2 & 0.2 & 0.1 & 0.1 & 0.1 \\
\hline Capital & 0.0 & 0.0 & 0.0 & 0.0 & 0.0 & 0.0 \\
\hline Total expenditure and net lending & 32.2 & 36.0 & 40.3 & 30.0 & 30.2 & 31.5 \\
\hline Total expenditure & 32.2 & 36.0 & 40.3 & 30.0 & 30.2 & 31.5 \\
\hline Current expenditure & 24.4 & 30.8 & 27.2 & 27.4 & 28.6 & 29.9 \\
\hline Expenditure on goods and services & 24.1 & 30.7 & 27.1 & 27.0 & 28.2 & 29.5 \\
\hline Wages and salaries & 14.1 & 15.2 & 15.5 & 15.8 & 16.7 & 16.5 \\
\hline Travel & 0.8 & 0.7 & 0.7 & 0.7 & 1.1 & 1.0 \\
\hline Other & 9.2 & 14.8 & 10.9 & 10.5 & 10.3 & 12.0 \\
\hline Subsidies & 0.3 & 0.1 & 0.1 & 0.2 & 0.2 & 0.2 \\
\hline Transfers & 0.0 & 0.0 & 0.0 & 0.1 & 0.2 & 0.2 \\
\hline Capital expenditure & 7.8 & 5.2 & 13.1 & 2.6 & 1.6 & 1.6 \\
\hline Acquisition of fixed capital & 1.0 & 0.7 & 0.9 & 0.8 & 1.0 & 0.7 \\
\hline Multi-purpose development projects & 6.8 & 4.4 & 4.8 & 1.8 & 0.6 & 0.9 \\
\hline Capital transfers & 0.0 & 0.0 & 7.5 & 0.0 & 0.0 & 0.0 \\
\hline Overall balance & 5.9 & 2.5 & -5.5 & -0.1 & 0.3 & -1.0 \\
\hline Current balance & 5.6 & -0.4 & 7.6 & 2.5 & 1.9 & 0.6 \\
\hline Capital balance & 0.3 & 2.9 & -13.1 & -2.6 & -1.6 & -1.6 \\
\hline
\end{tabular}

Source: Data provided by the FSM authorities; and Fund staff estimates.

1/ Government Financial Statistics (GFS) basis. Fiscal year ending September 30. 
Table 13. Federated States of Micronesia: Kosrae Government Finances, FY2002-07 1/

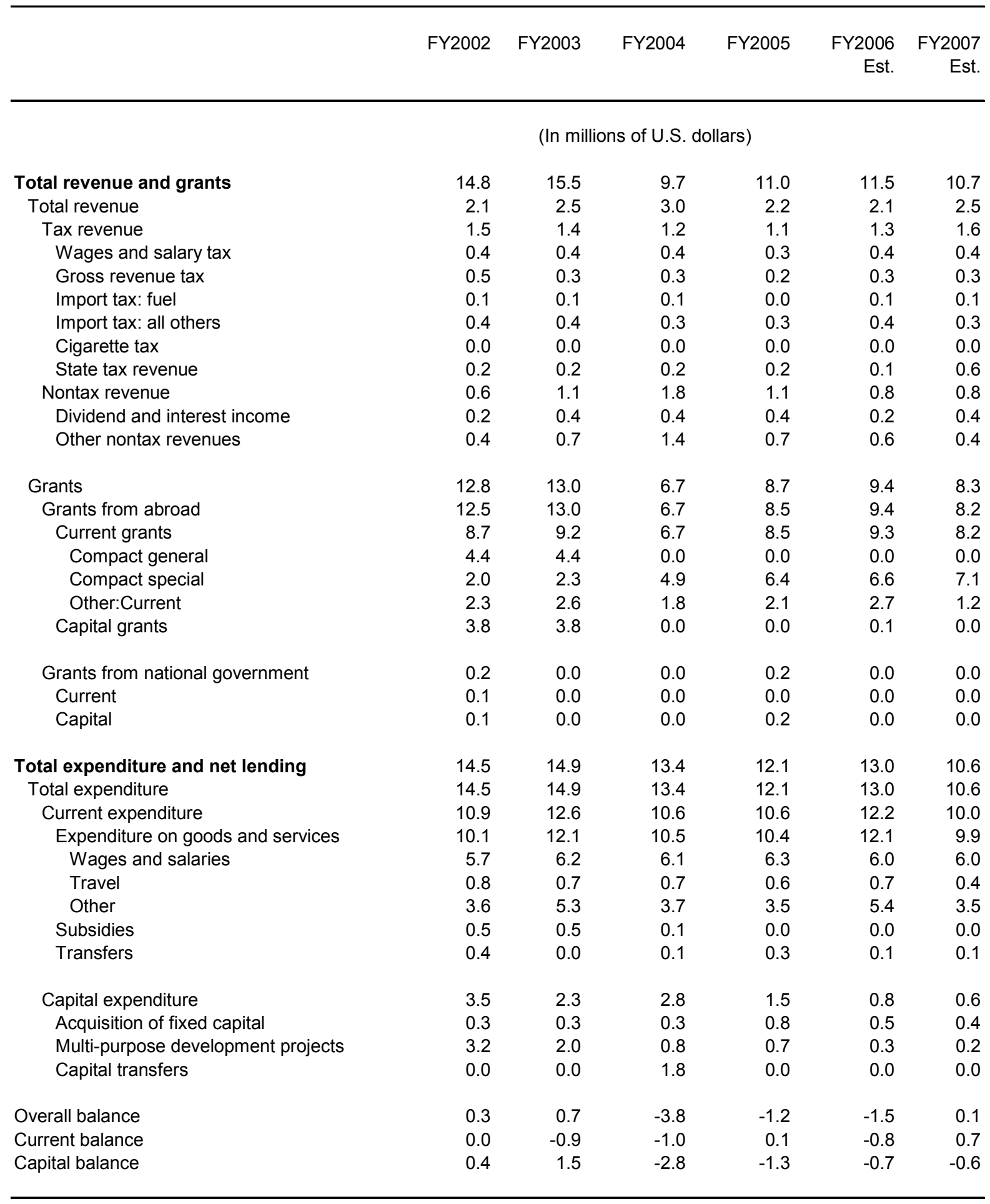

Source: Data provided by the FSM authorities; and Fund staff estimates.

1/ Government Financial Statistics (GFS) basis. Fiscal year ending September 30. 
Table 14. Federated States of Micronesia: Yap Government Finances, FY2002-07 1/

\begin{tabular}{|c|c|c|c|c|c|c|}
\hline & FY2002 & FY2003 & FY2004 & FY2005 & $\begin{array}{r}\text { FY2006 } \\
\text { Est. }\end{array}$ & $\begin{array}{r}\text { FY2007 } \\
\text { Est. }\end{array}$ \\
\hline & \multicolumn{6}{|c|}{ (In millions of U.S. dollars) } \\
\hline Total revenue and grants & 24.6 & 22.8 & 15.5 & 21.7 & 19.6 & 20.3 \\
\hline Total revenue & 6.4 & 4.4 & 5.1 & 5.3 & 5.7 & 4.9 \\
\hline Tax revenue & 3.2 & 2.7 & 3.9 & 4.0 & 4.0 & 3.5 \\
\hline Wages and salary tax & 0.7 & 0.5 & 1.0 & 0.4 & 0.6 & 0.5 \\
\hline Gross revenue tax & 0.7 & 0.4 & 0.7 & 0.7 & 0.6 & 0.6 \\
\hline Import tax: fuel & 0.1 & 0.0 & 0.0 & 0.1 & 0.1 & 0.1 \\
\hline Import tax: all others & 0.9 & 0.5 & 0.7 & 1.1 & 0.8 & 0.7 \\
\hline Cigarette tax & 0.0 & 0.0 & 0.0 & 0.0 & 0.4 & 0.2 \\
\hline State tax revenue & 0.8 & 1.3 & 1.4 & 1.7 & 1.6 & 1.5 \\
\hline Nontax revenue & 3.3 & 1.7 & 1.2 & 1.3 & 1.6 & 1.4 \\
\hline Dividend and interest income & 0.9 & 0.0 & 0.0 & 0.0 & 0.0 & 0.0 \\
\hline Other nontax revenues & 2.4 & 1.7 & 1.2 & 1.3 & 1.6 & 1.4 \\
\hline Grants & 18.1 & 18.4 & 10.4 & 16.3 & 13.9 & 15.4 \\
\hline Grants from abroad & 17.4 & 17.6 & 10.2 & 15.9 & 13.6 & 15.1 \\
\hline Current grants & 12.7 & 12.9 & 10.2 & 15.9 & 13.6 & 15.1 \\
\hline Compact general & 7.6 & 7.6 & 0.0 & 0.0 & 0.0 & 0.0 \\
\hline Compact special & 2.8 & 2.7 & 6.7 & 8.9 & 10.0 & 10.8 \\
\hline Other:Current & 2.3 & 2.6 & 3.5 & 7.0 & 3.6 & 4.3 \\
\hline Capital grants & 4.7 & 4.7 & 0.0 & 0.0 & 0.0 & 0.0 \\
\hline Grants from national government & 0.7 & 0.8 & 0.2 & 0.5 & 0.3 & 0.3 \\
\hline Current & 0.7 & 0.0 & 0.0 & 0.2 & 0.3 & 0.1 \\
\hline Capital & 0.0 & 0.8 & 0.2 & 0.2 & 0.0 & 0.2 \\
\hline Total expenditure and net lending & 16.9 & 17.0 & 22.7 & 25.3 & 21.5 & 23.9 \\
\hline Total expenditure & 16.9 & 17.0 & 22.7 & 25.3 & 21.5 & 23.9 \\
\hline Current expenditure & 15.0 & 15.3 & 15.5 & 20.6 & 20.0 & 21.6 \\
\hline Expenditure on goods and services & 14.0 & 14.7 & 15.0 & 20.3 & 19.4 & 20.1 \\
\hline Wages and salaries & 6.0 & 6.3 & 6.2 & 7.2 & 8.4 & 7.6 \\
\hline Travel & 1.4 & 0.8 & 0.9 & 1.0 & 1.0 & 1.2 \\
\hline Other & 6.6 & 7.6 & 7.9 & 12.1 & 10.0 & 11.3 \\
\hline Subsidies & 0.3 & 0.1 & 0.2 & 0.1 & 0.2 & 1.2 \\
\hline Transfers & 0.7 & 0.6 & 0.3 & 0.2 & 0.4 & 0.3 \\
\hline Capital expenditure & 1.8 & 1.7 & 7.3 & 4.7 & 1.5 & 2.2 \\
\hline Acquisition of fixed capital & 1.5 & 0.9 & 1.7 & 4.2 & 1.3 & 1.7 \\
\hline Multi-purpose development projects & 0.3 & 0.8 & 0.7 & 0.5 & 0.3 & 0.5 \\
\hline Capital transfers & 0.0 & 0.0 & 4.9 & 0.0 & 0.0 & 0.0 \\
\hline Overall balance & 7.7 & 5.7 & -7.2 & -3.6 & -2.3 & -3.8 \\
\hline Current balance & 4.8 & 2.0 & -0.2 & 0.9 & -0.8 & -1.7 \\
\hline Capital balance & 2.9 & 3.8 & -7.0 & -4.5 & -1.5 & -2.0 \\
\hline
\end{tabular}

Source: Data provided by the FSM authorities; and Fund staff estimates.

1/ Government Financial Statistics (GFS) basis. Fiscal year ending September 30. 
Table 15. Federated States of Micronesia: Chuuk Government Finances, FY2002-07 1/

\begin{tabular}{|c|c|c|c|c|c|c|}
\hline & FY2002 & $\begin{array}{r}\text { FY2003 } \\
\text { Est. }\end{array}$ & $\begin{array}{r}\text { FY2004 } \\
\text { Est. }\end{array}$ & $\begin{array}{r}\text { FY2005 } \\
\text { Est. }\end{array}$ & $\begin{array}{r}\text { FY2006 } \\
\text { Est. }\end{array}$ & $\begin{array}{r}\text { FY2007 } \\
\text { Est. }\end{array}$ \\
\hline & \multicolumn{6}{|c|}{ (In millions of U.S. dollars) } \\
\hline Total revenue and grants & 42.2 & 42.9 & 24.9 & 27.4 & 32.2 & 28.5 \\
\hline Total revenue & 5.6 & 6.6 & 6.5 & 5.4 & 7.0 & 5.2 \\
\hline Tax revenue & 4.4 & 5.6 & 5.2 & 4.8 & 4.9 & 4.1 \\
\hline Wages and salary tax & 0.9 & 1.1 & 1.1 & 0.9 & 0.9 & 0.6 \\
\hline Gross revenue tax & 0.9 & 0.9 & 0.9 & 0.8 & 0.8 & 0.7 \\
\hline Import tax: fuel & 0.2 & 0.2 & 0.2 & 0.2 & 0.2 & 0.1 \\
\hline Import tax: all others & 0.9 & 1.1 & 1.1 & 0.9 & 0.9 & 0.9 \\
\hline Cigarette tax & 0.0 & 0.0 & 0.0 & 0.0 & 0.2 & 0.0 \\
\hline State tax revenue & 1.5 & 2.3 & 2.0 & 2.0 & 2.0 & 1.9 \\
\hline Nontax revenue & 1.3 & 1.0 & 1.3 & 0.6 & 2.1 & 1.1 \\
\hline Dividend and interest income & 0.5 & 0.0 & 0.0 & 0.0 & 0.0 & 0.0 \\
\hline Other nontax revenues & 0.7 & 1.0 & 1.3 & 0.6 & 2.1 & 1.1 \\
\hline Grants & 36.6 & 36.4 & 18.4 & 22.0 & 25.3 & 23.2 \\
\hline Grants from abroad & 36.1 & 36.4 & 18.4 & 22.0 & 25.3 & 23.2 \\
\hline Current grants & 23.9 & 24.1 & 18.4 & 22.0 & 25.3 & 23.2 \\
\hline Compact general & 17.5 & 17.5 & 0.0 & 0.0 & 0.0 & 0.0 \\
\hline Compact special & 5.0 & 5.0 & 15.9 & 18.2 & 20.8 & 22.1 \\
\hline Other:Current & 1.4 & 1.6 & 2.4 & 3.7 & 4.5 & 1.1 \\
\hline Capital grants & 12.2 & 12.2 & 0.0 & 0.0 & 0.0 & 0.0 \\
\hline Compact CIP & 12.2 & 12.2 & 0.0 & 0.0 & 0.0 & 0.0 \\
\hline Other: capital & 0.0 & 0.0 & 0.0 & 0.0 & 0.0 & 0.0 \\
\hline Grants from national government & 0.5 & 0.0 & 0.0 & 0.0 & 0.0 & 0.0 \\
\hline Current & 0.5 & 0.0 & 0.0 & 0.0 & 0.0 & 0.0 \\
\hline Capital & 0.0 & 0.0 & 0.0 & 0.0 & 0.0 & 0.0 \\
\hline Total expenditure and net lending & 37.3 & 41.5 & 37.7 & 33.2 & 37.7 & 29.4 \\
\hline Total expenditure & 37.3 & 41.5 & 37.7 & 33.2 & 37.7 & 29.4 \\
\hline Current expenditure & 30.8 & 27.1 & 28.3 & 32.2 & 37.7 & 29.4 \\
\hline Expenditure on goods and services & 29.7 & 25.8 & 27.3 & 31.9 & 37.4 & 29.1 \\
\hline Wages and salaries & 19.5 & 18.5 & 0.0 & 0.0 & 0.0 & 0.0 \\
\hline Travel & 0.5 & 0.8 & 0.0 & 0.0 & 0.0 & 0.0 \\
\hline Other & 9.7 & 6.5 & 27.3 & 31.9 & 37.4 & 29.1 \\
\hline Subsidies & 1.0 & 1.2 & 1.0 & 0.4 & 0.2 & 0.2 \\
\hline Transfers & 0.0 & 0.1 & 0.0 & 0.0 & 0.1 & 0.0 \\
\hline Capital expenditure & 6.5 & 14.4 & 9.3 & 1.0 & 0.0 & 0.0 \\
\hline Acquisition of fixed capital & 0.5 & 9.7 & 0.0 & 0.0 & 0.0 & 0.0 \\
\hline Multi-purpose development projects & 6.0 & 4.7 & 3.3 & 1.0 & 0.0 & 0.0 \\
\hline Capital transfers & 0.0 & 0.0 & 6.1 & 0.0 & 0.0 & 0.0 \\
\hline Overall balance & 4.9 & 1.5 & -12.8 & -5.8 & -5.7 & -0.9 \\
\hline Current balance & -0.8 & 3.6 & -3.4 & -4.9 & -5.6 & -0.9 \\
\hline Capital balance & 5.7 & -2.2 & -9.3 & -1.0 & 0.0 & 0.0 \\
\hline
\end{tabular}

Source: Data provided by the FSM authorities; and Fund staff estimates.

1/ Government Financial Statistics (GFS) basis. Fiscal year ending September 30. 
Table 16. Federated States of Micronesia: Banking Survey, FY2002-07 1/

(In millions of U.S. dollars; end of period)

\begin{tabular}{|c|c|c|c|c|c|c|}
\hline & 2002 & 2003 & 2004 & 2005 & 2006 & 2007 \\
\hline Assets & 128.3 & 137.1 & 131.2 & 128.4 & 132.4 & 139.4 \\
\hline Liquid Assets & 83.5 & 107.7 & 104.4 & 97.2 & 96.2 & 97.5 \\
\hline Cash and due from local banks & 5.2 & 3.1 & 4.0 & 3.6 & 4.2 & 4.1 \\
\hline Foreign assets - due from banks abroad & 78.3 & 104.6 & 100.5 & 93.6 & 92.0 & 93.5 \\
\hline Loans & 39.1 & 24.0 & 21.3 & 25.7 & 30.0 & 35.3 \\
\hline Commercial loans & 21.0 & 10.1 & 8.8 & 10.7 & 14.6 & 19.4 \\
\hline Consumer loans & 18.1 & 13.9 & 12.5 & 14.9 & 15.4 & 15.9 \\
\hline Other assets & 5.7 & 5.4 & 5.5 & 5.6 & 6.2 & 6.6 \\
\hline Liabilities & 128.3 & 137.1 & 131.2 & 128.4 & 132.4 & 139.4 \\
\hline Deposits & 112.0 & 119.8 & 115.4 & 111.4 & 113.7 & 119.5 \\
\hline Demand & 25.5 & 28.1 & 28.0 & 28.3 & 27.6 & 32.8 \\
\hline Savings & 44.7 & 60.2 & 56.6 & 50.3 & 51.7 & 47.2 \\
\hline Time & 41.7 & 31.4 & 30.7 & 32.8 & 34.4 & 39.4 \\
\hline Other & 0.1 & 0.1 & 0.1 & 0.1 & 0.1 & 0.1 \\
\hline Liabilities and capital & 16.3 & 17.3 & 15.8 & 17.0 & 18.7 & 19.9 \\
\hline & \multicolumn{6}{|c|}{ (In percent) } \\
\hline \multicolumn{7}{|l|}{ Memorandum items: } \\
\hline Loan/deposit ratio & 34.9 & 20.0 & 18.5 & 23.0 & 26.4 & 29.5 \\
\hline Commercial loan share of total & 53.8 & 42.1 & 41.2 & 41.8 & 48.6 & 55.0 \\
\hline Consumer loan share of total & 46.2 & 57.9 & 58.8 & 58.2 & 51.4 & 45.0 \\
\hline Deposits annual rate of change & -7.9 & 6.9 & -3.7 & -3.4 & 2.1 & 5.1 \\
\hline Loans annual rate of change & -24.9 & -38.7 & -11.0 & 20.4 & 16.9 & 17.7 \\
\hline Commercial loans annual rate of change & 15.0 & -52.0 & -13.0 & 22.1 & 35.9 & 33.2 \\
\hline Consumer loans annual rate of change & -46.5 & -23.3 & -9.6 & 19.2 & 3.2 & 2.9 \\
\hline
\end{tabular}

Source: FSM Banking Board.

$1 /$ On a fiscal year basis. 
Table 17. Federated States of Micronesia: Interest Rates of Deposit Money Banks, 2001-08 1/

(In percent per annum; end of period)

\begin{tabular}{|c|c|c|c|c|c|c|c|c|}
\hline & 2001 & 2002 & 2003 & 2004 & 2005 & 2006 & 2007 & 2008 \\
\hline \multicolumn{9}{|l|}{ Deposit rates 2/ } \\
\hline Savings deposits & 1.7 & 1.3 & 0.9 & 0.9 & 1.1 & 1.9 & $\ldots$ & $\ldots$ \\
\hline CDs & 1.5 & 1.3 & 0.9 & 1.3 & 2.4 & 2.5 & $\ldots$ & $\ldots$ \\
\hline Other deposits & 3.1 & 1.3 & 1.0 & 1.0 & 1.5 & 2.1 & $\ldots$ & $\ldots$ \\
\hline \multicolumn{9}{|l|}{ Loan rates $2 /$} \\
\hline Consumer loans & 15.3 & 15.0 & 15.0 & 16.5 & 16.5 & 13.4 & 14.4 & 14.4 \\
\hline Commercial loans & 5.9 & 4.8 & 7.0 & 7.5 & 9.5 & 8.9 & 9.2 & 8.6 \\
\hline
\end{tabular}

Source: Data provided by the FSM authorities.

1/ On calendar year basis. Data for 2008 are for August.

2/ Average of rates offered and charged by deposit money banks. 
Table 18. Federated States of Micronesia: Balance of Payments, FY2002-06

(In millions of U.S. dollars)

\begin{tabular}{|c|c|c|c|c|c|}
\hline & FY2002 & FY2003 & FY2004 & FY2005 & $\begin{array}{r}\text { FY2006 } \\
\text { Est. }\end{array}$ \\
\hline Trade balance & -87.7 & -96.2 & -120.3 & -122.2 & -128.9 \\
\hline Exports, f.o.b. & 23.8 & 24.0 & 17.0 & 18.5 & 17.0 \\
\hline Imports, f.o.b. 1/ & -111.5 & -120.2 & -137.3 & -140.7 & -145.9 \\
\hline Petroleum products & -14.9 & -14.4 & -17.2 & -21.7 & -27.0 \\
\hline Services account & -36.1 & -36.0 & -37.3 & -38.2 & -37.3 \\
\hline Receipts & 18.9 & 18.6 & 18.2 & 18.5 & 20.1 \\
\hline Travel & 16.8 & 16.7 & 16.5 & 17.1 & 18.3 \\
\hline Communications (net) & 2.1 & 1.9 & 1.5 & 1.3 & 1.8 \\
\hline Other & 0.1 & 0.1 & 0.3 & 0.1 & 0.0 \\
\hline Payments & -55.0 & -54.7 & -55.5 & -56.7 & -57.5 \\
\hline Freight and insurance & -19.7 & -21.2 & -24.2 & -24.8 & -25.8 \\
\hline Transportation & -12.8 & -11.4 & -11.4 & -12.1 & -12.3 \\
\hline Travel & -5.3 & -5.5 & -5.3 & -5.5 & -5.7 \\
\hline Other & -17.3 & -16.6 & -14.5 & -14.3 & -13.7 \\
\hline Income, net & 10.4 & 8.9 & 13.7 & 16.2 & 15.2 \\
\hline Receipts & 16.4 & 15.6 & 20.1 & 21.5 & 20.5 \\
\hline Fishing rights fees & 10.6 & 11.8 & 12.1 & 13.3 & 13.3 \\
\hline Interest dividend income & 5.8 & 3.8 & 8.1 & 8.2 & 7.2 \\
\hline Payments & -6.0 & -6.7 & -6.4 & -5.3 & -5.3 \\
\hline Foreign workers earnings & -3.0 & -3.5 & -3.3 & -2.3 & -2.3 \\
\hline Interest payments & -1.7 & -1.8 & -1.8 & -1.6 & -1.6 \\
\hline Dividends & -1.3 & -1.4 & -1.3 & -1.4 & -1.4 \\
\hline Unrequited transfers & 104.3 & 123.0 & 99.1 & 115.2 & 112.7 \\
\hline Private & 2.3 & 2.3 & 2.5 & 3.4 & 5.3 \\
\hline Inflows & 4.8 & 4.9 & 5.0 & 6.0 & 8.0 \\
\hline Outflows & -2.5 & -2.6 & -2.5 & -2.6 & -2.7 \\
\hline Official & 101.9 & 120.6 & 96.6 & 111.8 & 107.3 \\
\hline Compact funds & 65.9 & 66.6 & 52.1 & 56.0 & 60.1 \\
\hline Other & 36.0 & 54.0 & 44.5 & 55.9 & 47.3 \\
\hline Current account balance & -9.1 & -0.4 & -44.7 & -29.1 & -38.4 \\
\hline Capital and financial account & 38.4 & 17.0 & -13.9 & 20.1 & 18.9 \\
\hline Capital transfers & 39.1 & 39.8 & -21.6 & 7.1 & 16.2 \\
\hline Capital transfers, official & 31.8 & 32.2 & -27.7 & 0.0 & 7.2 \\
\hline Capital transfers, in-kind & 7.3 & 7.6 & 6.1 & 7.1 & 9.1 \\
\hline Financial account & -0.7 & -22.7 & 7.7 & 13.1 & 2.7 \\
\hline Short term, net & -0.8 & -25.0 & 7.5 & 12.0 & 0.8 \\
\hline Medium term, net & 0.1 & 2.2 & 0.3 & 1.1 & 1.9 \\
\hline Inflows & 0.7 & 2.9 & 1.1 & 1.9 & 3.7 \\
\hline Medium-term notes (MTN) & 0.0 & 0.0 & 0.0 & 0.0 & 0.0 \\
\hline Other borrowing & 0.7 & 2.9 & 1.1 & 1.9 & 3.7 \\
\hline Outflows & -0.6 & -0.6 & -0.8 & -0.9 & -1.8 \\
\hline MTN amortization & 0.0 & 0.0 & 0.0 & 0.0 & 0.0 \\
\hline Other amortization & -0.6 & -0.6 & -0.8 & -0.9 & -1.8 \\
\hline Overall balance & 29.3 & 16.6 & -58.6 & -8.9 & -19.5 \\
\hline
\end{tabular}

Source: Data provided by the FSM authorities. 
Table 19. Federated States of Micronesia: External Debt and Debt Service Obligations, FY2001-07

(In millions of U.S. dollars)

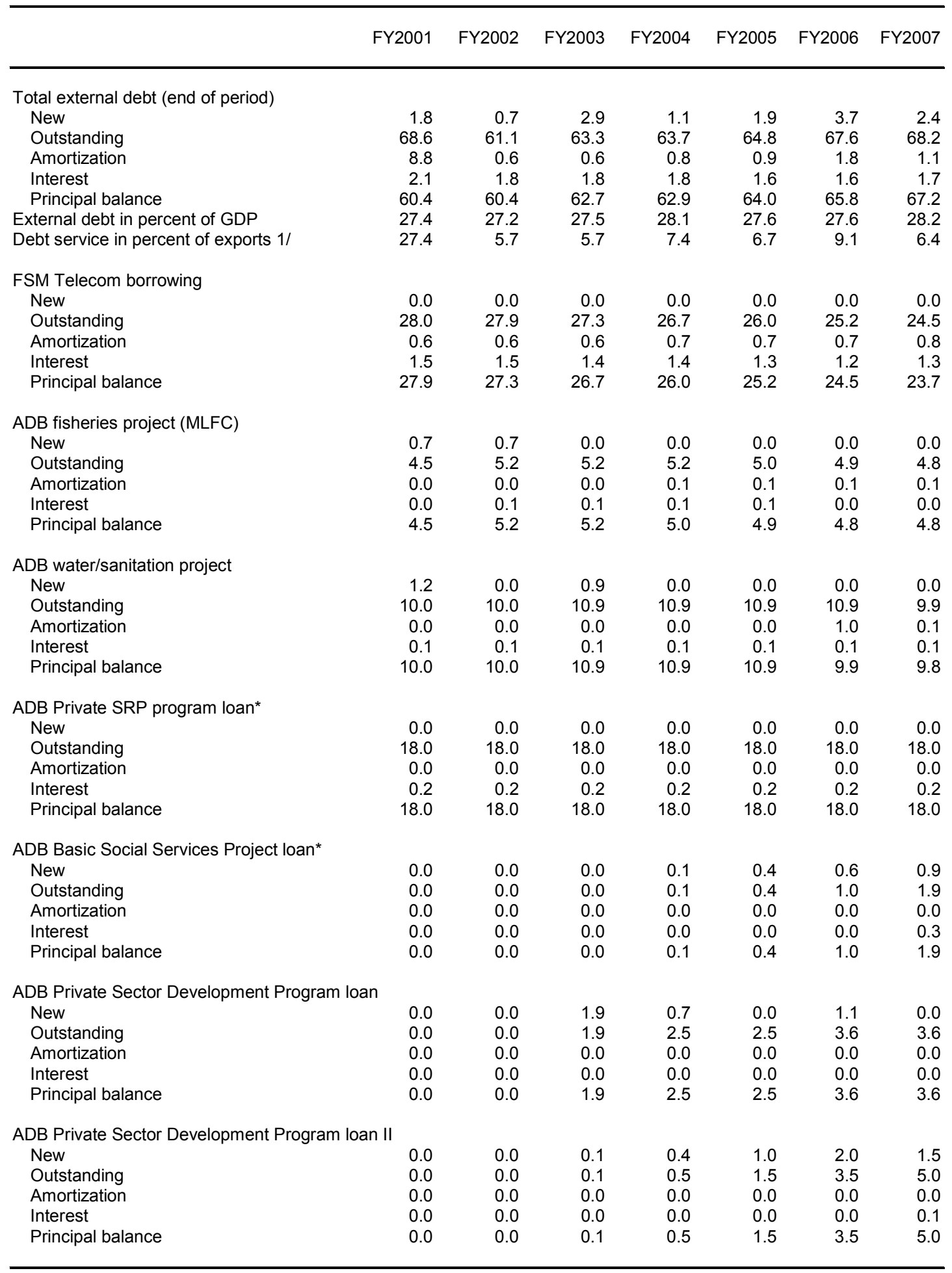

Source: Data provided by the FSM authorities.

$1 /$ Export of goods and services. 